\title{
Lipid-associated PML structures assemble nuclear lipid droplets containing CCT $\alpha$ and Lipin1
}

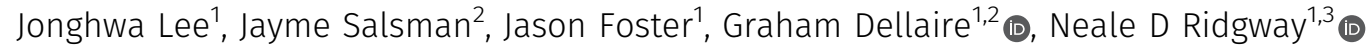

\begin{abstract}
Nuclear lipid droplets (nLDs) form on the inner nuclear membrane by a mechanism involving promyelocytic leukemia (PML), the protein scaffold of PML nuclear bodies. We report that PML structures on nLDs in oleate-treated U2OS cells, referred to as lipid-associated PML structures (LAPS), differ from canonical PML nuclear bodies by the relative absence of SUM01, SP100, and DAXX. These nLDs were also enriched in CTP:phosphocholine cytidylyltransferase $\alpha$ (CCT $\alpha)$, the phosphatidic acid phosphatase Lipin1, and DAG. Translocation of CCT $\alpha$ onto nLDs was mediated by its $\alpha$-helical M-domain but was not correlated with its activator DAG. High-resolution imaging revealed that CCT $\alpha$ and LAPS occupied distinct polarized regions on nLDs. PML knockout U2OS (PML KO) cells lacking LAPS had a 40-50\% reduction in nLDs with associated CCT $\alpha$, and residual nLDs were almost devoid of Lipin1 and DAG. As a result, phosphatidylcholine and triacylglycerol synthesis was inhibited in PML KO cells. We conclude that in response to excess exogenous fatty acids, LAPS are required to assemble nLDs that are competent to recruit CCT $\alpha$ and Lipin1.
\end{abstract}

Dol 10.26508/Isa.202000751 | Received 22 April 2020 | Revised 13 May 2020 | Accepted 14 May 2020 | Published online 27 May 2020

\section{Introduction}

Lipid droplets (LDS) are cellular organelles composed of a core of triacylglycerol (TAG) and cholesterol ester (CE) surrounded by a monolayer of phospholipids and associated proteins. In response to nutrient and hormonal signals, fatty acids and cholesterol are stored in or released from LDs to provide energy and biosynthetic precursors as well as buffer the cell from fatty acid toxicity (Sztalryd \& Brasaemle, 2017). The largest neutral lipid storage depot is in adipocytes but hepatocytes, enterocytes, and macrophages also have the capacity for short-term storage and release of fatty acids from LDs. Accumulation of LDs in tissues, caused by an imbalance between lipid storage and hydrolysis, is linked to pathological conditions such as hepatosteatosis, obesity, and lipodystrophy (Walther \& Farese, 2012).

LDs are proposed to form in the ER by a process that requires the coordinated synthesis of TAG and phospholipids (Henne et al, 2018).
TAG initially forms a "lens" in the ER bilayer, expands, and pinches off into the cytoplasm and is coated with ER-derived phospholipids, which regulate surface tension and the storage capacity of LDS (Krahmer et al, 2011). Phosphatidylcholine (PC) is the most significant component of the surface monolayer of LDs (Tauchi-Sato et al, 2002; Bartz et al, 2007), and de novo PC synthesis by the cytidine diphosphate (CDP)-choline pathway in the ER is required for LD biogenesis (Krahmer et al, 2011; Aitchison et al, 2015). In mammalian cells, the CDP-choline pathway is regulated by CTP:phosphocholine cytidylyltransferase (CCT) $\alpha$ and $\beta$ isoforms that are activated by translocation to nuclear and cytoplasmic membranes, respectively, in response to the content of PC and specific lipid activators, such as fatty acids or the type II conical-shaped lipids DAG and phosphatidylethanolamine (PE) (Arnold \& Cornell, 1996; Arnold et al, 1997; Xie et al, 2004). During LD biogenesis in fatty acid-treated cells (Lagace \& Ridgway, 2005; Gehrig et al, 2009) and during adipocyte differentiation (Aitchison et al, 2015), PC synthesis was increased by $\mathrm{CCT} \alpha$ translocation from the nucleoplasm to the inner nuclear membrane (INM). In oleate-treated insect cells, ectopically expressed CCT $\alpha$ and the insect homologue CCT1 translocated from the nucleus to the surface of cytoplasmic LDs (cLDs) resulting in increased PC synthesis to facilitate LD expansion and TAG storage (Guo et al, 2008; Krahmer et al, 2011; Payne et al, 2014). In contrast, mammalian CCT $\alpha$ can exit the nucleus under some conditions (Northwood et al, 1999; Gehrig et al, 2009) but does not localize to the surface of cLDs in adipocytes and other cultured cells (Aitchison et al, 2015; Haider et al, 2018).

In addition to the INM, CCT $\alpha$ translocation to nuclear LDs (nLDs) can activate PC synthesis in oleate-treated Huh7 hepatoma cells (Soltysik et al, 2019). nLDs account for $\approx 10 \%$ of the total cellular LD pool in liver sections and hepatocytes and have a unique lipid and protein composition (Uzbekov \& Roingeard, 2013; Lagrutta et al, 2017). In hepatocytes, nLDs can arise from TAG-rich droplets in the ER lumen that are precursors for very low density lipoprotein (VLDL) assembly (Soltysik et al, 2019). Instead of being incorporated into secreted VLDL, ER luminal LDs migrate into invaginations of the INM, termed as the type I nucleoplasmic reticulum (NR), and are released as nascent nLDs into the nucleoplasm where they could increase in size by fusion with other nLDs or by de novo TAG

\footnotetext{
${ }^{1}$ Department of Biochemistry and Molecular Biology, Dalhousie University, Halifax, Canada ${ }^{2}$ Department of Pathology, Dalhousie University, Halifax, Canada ${ }^{3}$ Department of Pediatrics, Dalhousie University, Halifax, Canada
}

Correspondence: dellaire@dal.ca; nridgway@dal.ca 
synthesis (Ohsaki et al, 2016; Soltysik et al, 2019). During or after release into the nucleoplasm, nLDs associate with promyelocytic leukemia (PML) protein, in what were at the time referred to as PML nuclear bodies (PML NBs) (Ohsaki et al, 2016). PML NBs are dynamic subnuclear domains that regulate gene expression and stress responses and are strongly associated with proteins modified by the small ubiquitin modifier 1 (SUMO1) (Dellaire \& Bazett-Jones, 2004; Ching et al, 2005; Van Damme et al, 2010), the most prominent being the SP100 nuclear antigen and the death-associated domain protein 6 (DAXX) (Zuber et al, 1995; Ishov et al, 1999). However, of the seven PML isoforms, only PML-II was associated with nLDs and required for their formation (Ohsaki et al, 2016). Moreover, the presence of CCT $\alpha$ and perilipin-3 on nLDs suggests that these lipidassociated PML-containing domains can regulate lipid synthesis (Soltysik et al, 2019), possibly to generate a metabolic signal to accommodate the uptake and storage of excess fatty acids.

Here, we report that the PML structures on $n L D$ s in oleate-treated U2OS cells are deficient of SUMO1, SP100, and DAXX, proteins that are commonly associated with PML NBs. Therefore, to distinguish these structures from canonical PML NBs and reflect their association with nLDs and the lipid biosynthetic enzymes CCT $\alpha$ and Lipin1, we have designated them as lipid-associated PML structures (LAPS). Disrupting LAPS by CRISPR/Cas9 knockout of the PML gene in human U2OS osteosarcoma cells (PML KO) resulted in fewer nLDS that were deficient in CCT $\alpha$, Lipin1, and DAG, resulting in decreased PC and TAG synthesis. These findings support the concept that LAPS are required for assembly of $n L D s$ and nuclear lipid synthesis and that nLDs could provide a regulatory platform to orchestrate the cellular response to excess fatty acid uptake and storage.

\section{Results}

\section{nLDs associate with non-canonical LAPS}

Similar to hepatoma cells, U2OS osteosarcoma cells contain abundant nLDs that are associated with mCherry-PML-II (Ohsaki et al, 2016). As further evidence of their nucleoplasmic localization, nLDs in oleate-treated U2OS cells have endogenous PML and CCT $\alpha$ on their surface (Fig S1), and PML-positive nLDs were not encapsulated by the nuclear envelope (NE) based on immunostaining for emerin (Fig S2). Thus, U2OS cells offer an alternate and complementary model to investigate the structure and function of nLDs. Initially, we investigated whether the PML structures on nLDs differ from canonical PML NB by immunostaining control and oleatetreated U2OS cells for the PML NB-associated proteins SUMO1, SP100, and DAXX (Figs 1, S3, and S4). U2OS cells contain numerous PML NBs that are all positive for SUMO1, consistent with the essential role of SUMOylation in PML NB assembly and function (Zhong et al, 2000) (Fig 1A). Oleate treatment caused the loss of SUMO1-positive PML NB puncta and the appearance of PMLpositive nLDs with different levels of SUMO1 expression (Fig 1B). When quantified, a weak ( $<50 \%$ of PML signal intensity) or nonexistent SUMO1 signal was detected in $75 \%$ of PML-positive nLDS (Fig 1C). In addition, DAXX and SP100, proteins whose interaction with canonical PML NBs is SUMO-dependent (Ishov et al, 1999) or constitutive (Sternsdorf et al, 1997), respectively, were strongly localized to PML NBs in untreated cells but weakly associated or absent in $80 \%$ of PML-positive nLDs in oleate-treated U2OS cells (Figs 1C, S3, and S4). Since the PML structures on nLDs are part of a large lipid complex and relatively devoid of canonical PML NB proteins, we propose that they be designated as LAPS.

To investigate how LAPS influence the composition and biogenesis of nLDs, we used U2OS cells in which the PML gene was knocked out (PML KO) by CRISPR/Cas9 gene editing leading to loss of expression of all PML isoforms (Attwood et al, 2019). CCT $\alpha$ or PML expression in U2OS cells was unchanged by oleate treatment, and CCT $\alpha$ expression was unaffected by PML KO (Fig 2A). In oleatetreated U2OS cells, CCT $\alpha$ was expressed in the nucleoplasm and on the surface of nLDs (Fig 2B), whereas, in PML KO cells, there were fewer BODIPY- and CCT $\alpha$-positive $n L D s$, and CCT $\alpha$ was partially localize to the NE. Compared with control cells, the number of cLDs in PML KO cells was reduced slightly (Fig $2 \mathrm{C}$ ). However, PML KO cells had a significant reduction in the number of $n L D$ per cell (Fig 2D) and the percentage of CCT $\alpha$-positive nLDs (Fig 2E). The cross-sectional area of CLDs in PML KO cells was similar to controls (Fig 2F), but there was a significant shift in the distribution toward small nLDs in PML KO cells (Fig 2G) that was reflected in a $40 \%$ decrease in average area (Fig $2 \mathrm{H}$ ). CCT $\alpha$-positive nLDs in control and PML KO cells were similar in size, suggesting the enzyme preferentially associates with larger nLDs that are formed by a PML-independent mechanism.

To identify the PML isoform involved in $\mathrm{nLD}$ formation in U2OS cells, PML KO cells were individually transfected with GFP-tagged version of the seven PML isoforms. In agreement with results in Huh7 cells (Ohsaki et al, 2016), GFP-PML-II associated with nLDs and increased their abundance in PML KO cells, whereas other PML isoforms did not associate or correct the PML KO phenotype (Fig S5A and $B$, results not shown). GFP-PML-II expression in PML KO cells significantly increased the number and size of nLDs to the level observed in wild-type U2OS cells (Fig S5C and D) and also restored the slight reduction in these parameters for CLDs (Fig S5E and F). The specific requirement for PML-II in $\mathrm{nLD}$ formation is further evidence that LAPS are a unique nuclear PML subdomain.

\section{CCT $\alpha$ and LAPS localize to discrete regions of nLDs}

CCT $\alpha$ associates with membranes via its amphipathic $\alpha$-helical M-domain, which is antagonized by phosphorylation of 16 serine, threonine, and tyrosine residues in the adjacent P-domain (Fig 3A) (Cornell \& Ridgway, 2015). The binding of CCT $\alpha$ to LDs involves large hydrophobic side chains in the M-domain that insert into voids in the phospholipid monolayer (Prevost et al, 2018). To determine if the $\mathrm{M}$ - and $\mathrm{P}$-domains regulate binding to $\mathrm{nLDs}$, CCT $\alpha$ with truncations and point mutations in these domains was expressed in oleate-treated U2OS cells (Fig $3 \mathrm{~A}$ and B), and localization on nLDs was quantified by immunofluorescence microscopy (Fig 3C and D). $\mathrm{CCT} \alpha$ localization to $\mathrm{nLDs}$ was completely prevented by mutation of eight lysine residues in the $\mathrm{M}$-domain (CCT $\alpha$-8KQ) that form electrostatic interactions with membrane lipids (Johnson et al, 2003). Conversely, CCT $\alpha-3 E Q$, an M-domain mutant with enhanced membrane association (Johnson et al, 2003), was localized to $\mathrm{nLDs}$ as well as the NE. Deletion of the P-domain (CCT $\alpha-\Delta \mathrm{P})$ and a dephosphorylated mimic with 16 serine residues mutated to 


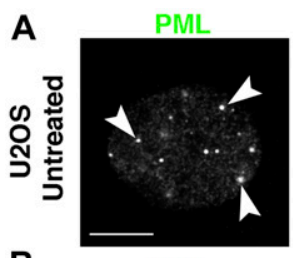

B

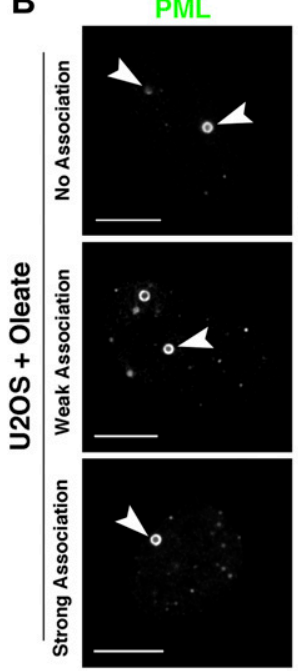

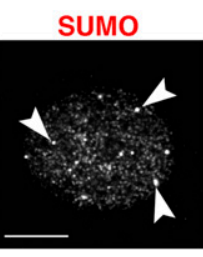

SUMO
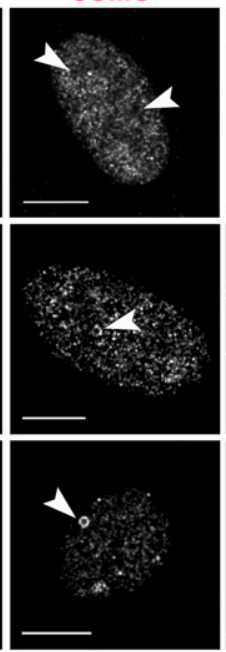

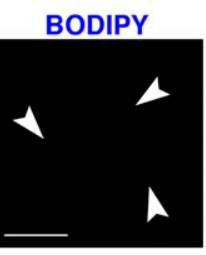

BODIPY
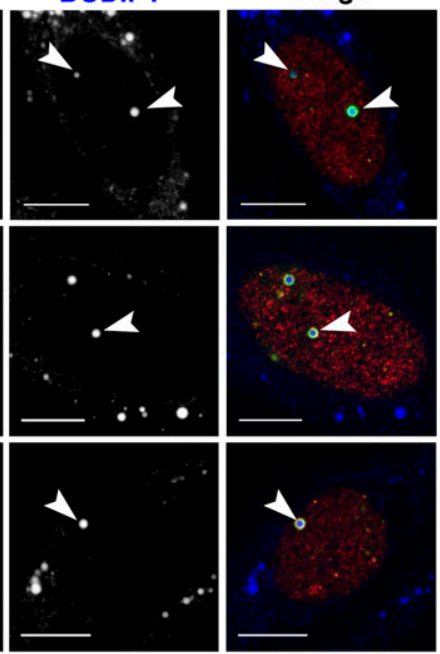

C PML NB component association with LAP domains
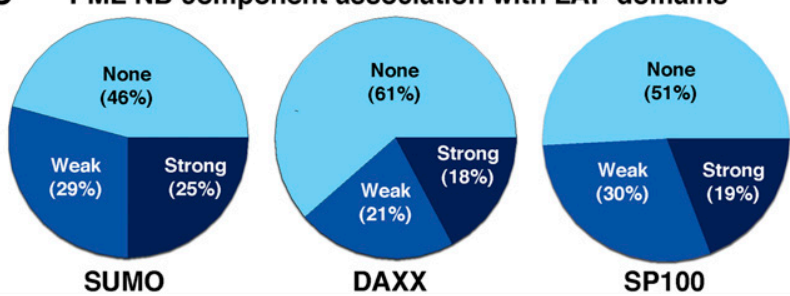

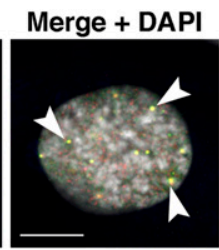

Merge + DAPI
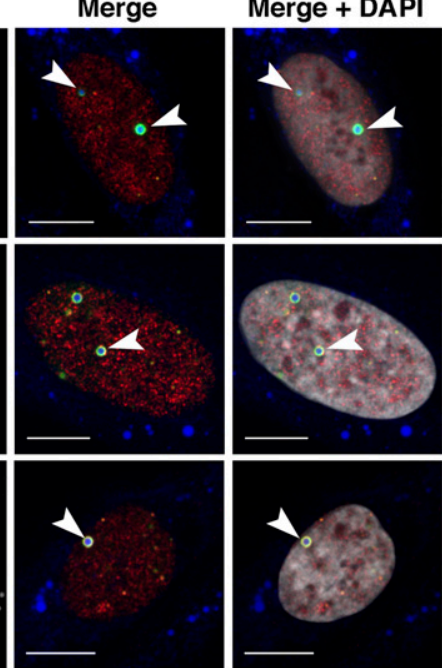

Figure 1. Lipid-associated PML structures (LAPS) on nuclear lipid droplets (nLDs) are deficient in PML nuclear body (NB) resident proteins.

(A, B) U2OS cells were untreated (panel A) or incubated with oleate $(400 \mu \mathrm{M})$ for $24 \mathrm{~h}$ (panel B) before immunostaining for PML (green) and SUMO1 (red) and imaging by confocal microscopy. LDs and nuclei were visualized with BODIPY 493/503 and DAPI, respectively (bar, $10 \mu \mathrm{m}$ ). (B) Examples of SUMO1 association (none, weak, or strong) with PML-positive nLDs are shown (panel B). (c) The intensity of SUMO1, DAXX, and SP100 association with PML-positive nLDs was scored as none, weak, or strong (described in the Materials and Methods section) for at least 100-150 nLDs from at least 50-100 cells. Examples of DAXX and SP100 localization with PML-positive nLDs are shown in Figs S3 and S4. alanine (CCT $\alpha-16 S A)$ were strongly associated with nLDs, whereas the phosphorylated mimic with 16 serine residues mutated to glutamate (CCT $\alpha$-16SE) was not detected on nLDS. Catalytic dead CCT $\alpha-K 122 A$ was localized on nLDs similarly to the wild type. These results indicate that interaction of $\mathrm{CCT} \alpha$ with the nLD phospholipid monolayer is mediated by electrostatic interactions with the M-domain and antagonized by phosphorylation of the P-domain.

Next, we used spinning disk confocal 3D and super-resolution radial fluctuation (SRRF) (Gustafsson et al, 2016) imaging to determine how endogenous CCT $\alpha$ and LAPS are organized on the surface of nLDs (Fig 4). 3D reconstruction of a typical nLD revealed a lipid body (BODIPY) located close to the basal surface of the INM (Fig 4A, a, b, and C). CCT $\alpha$ coated most of the nLD but was absent from the top of the particle (Fig 4A, d), which was occupied by a "cap" of PML protein (Fig 4A, e and f). SRRF imaging of this nLD in a cross section where CCT $\alpha$ and PML intersect (indicted by the yellow line in Fig 4A, b-f) revealed a punctate distribution (Fig 4B and C) consistent with each protein occupying non-overlapping, interdigitated regions on the nLD surface (error and resolution analysis using NanoJ-SQUIRREL [Culley et al, 2018] for this image is shown in Fig S6). Additional 3D renderings of eight nLDs are shown in Fig S7. In the case of smaller nLDs (Fig S7A, object 1; Fig S7C, objects 5 and 6),
PML formed a patch on the surface that minimally overlapped with CCT $\alpha$. Similar to Fig 4, large nLDs displayed a polarized PML patch (Fig S7A, object 2; Fig S7B, object 3). More infrequent were large nLDs with PML coating much of the surface and reduced CCT $\alpha$ association (Fig S7B, object 4). In summary, LAPS generally occupy only part of the surface of an nLD and have limited overlap with CCT $\alpha$, which more strongly associates with large nLDs via its M-domain.

\section{LAPS regulate the DAG and Lipin1 content of nLDs}

In addition to being a precursor for the TAG and PC that is incorporated into LDs, DAG also stimulates translocation and activation of CCT $\alpha$ on membranes (Arnold et al, 1997). Thus, we investigated whether CCT $\alpha$ interaction with nLDs could be regulated by DAG as a mechanism to coordinate PC and TAG synthesis. Initially, we visualized DAG in cells cultured with or without oleate for $24 \mathrm{~h}$ using the DAG biosensor GFP-C1(2) $\delta$ (Codazzi et al, 2001) (Fig 5). In untreated wild-type and U2OS PML KO cells, the DAG biosensor was localized on reticular and punctate structures in the cytoplasm (Fig 5A). Exposure of wild-type U2OS cells to oleate resulted in the appearance of the DAG biosensor on dispersed cytoplasmic 
A

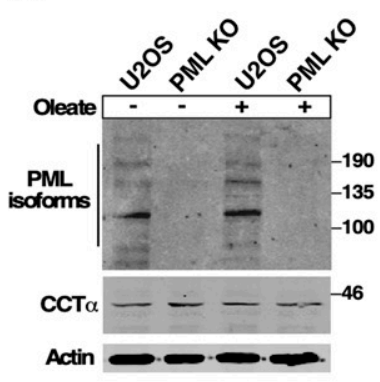

B
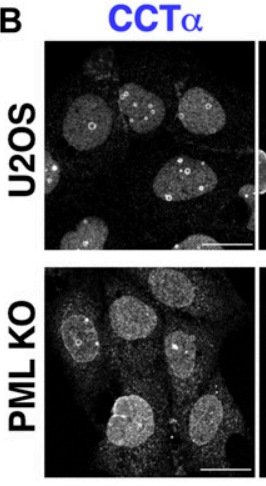

D

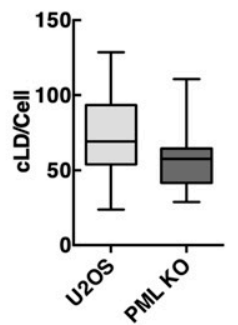

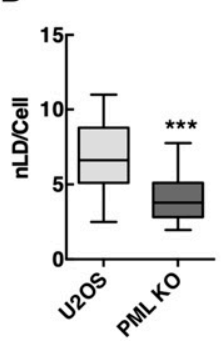

E

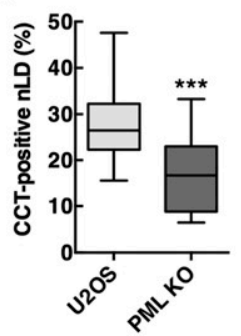

LMNAC
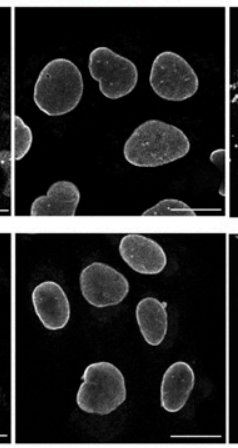
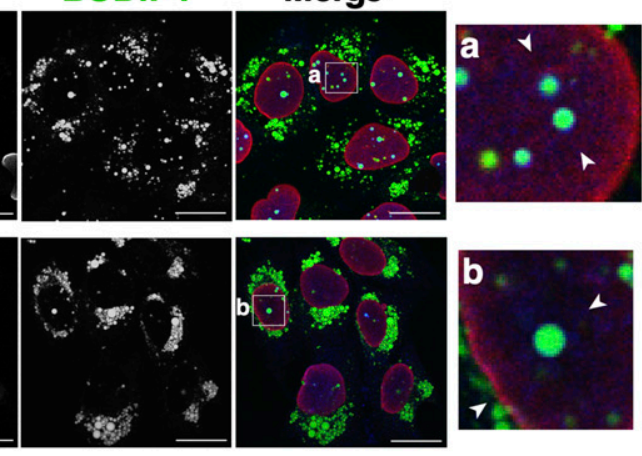

F

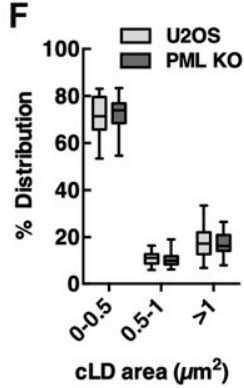

Merge

G

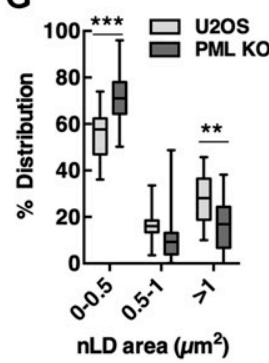

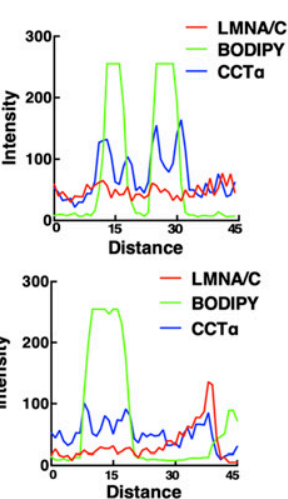

H

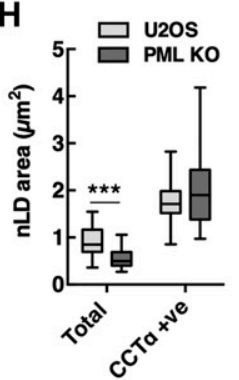

Figure 2. Lipid-associated PML structures regulate biogenesis of nuclear lipid droplets (nLDs) and association of CCTa.

(A) Lysates of U2OS and PML KO cells that were untreated or incubated with oleate (400 $\mu \mathrm{M})$ for $24 \mathrm{~h}$ were immunoblotted with PML-, CCT $\alpha$-, and actin-specific antibodies. (B) U2OS and PML KO cells were immunostained for CCT $\alpha$ and LMNA/C, and LDs were visualized with BODIPY 493/503 (bar, $20 \mu \mathrm{m}$ ). Arrows in magnified panels (a) and (b) indicate the regions for RGB line scan plots showing the localization of CCTa on the surface of nLDs. (C, D) Quantitation of cytoplasmic (cLDs) and nLDs in cells treated with oleate $(400 \mu \mathrm{M})$ for $24 \mathrm{~h}$. (E) Quantitation of CCT $\alpha$-positive $n$ LDs in oleate-treated cells. (F, G) The size distribution of CLDs (panel F) and nLDs (panel G) in oleate-treated cells was determined by cross-sectional area binning. $(\mathrm{H})$ Average cross-sectional area of total and CCTa-positive nLDs was quantified in oleate-treated cells. In panels (C, D, E, F, G, H), results are presented as box and whisker plots showing the mean and $5^{\text {th }}-95^{\text {th }}$ percentile for analysis of 50-100 cells from three separate experiments. Significance was determined by two-tailed $t$ test compared with matched U2OS cell controls $\left({ }^{\star \star} P<0.01\right.$; $\left.{ }^{\star \star \star} P<0.001\right)$.

structures that did not correspond to CLDs, and occasionally on LipidTox Red-positive nLDs (Fig 5B, a-c). In contrast, the DAG biosensor was strongly associated with CLDs in PML KO cells and not observed on nLDs (Fig 5C, d-f).
To better assess the distribution of DAG on nLDs, a nuclearlocalized DAG biosensor (nGFP-DAG) was made by inserting a tandem NLS into GFP-C1(2) $\delta$. When transiently expressed in oleatetreated U2OS cells, nGFP-DAG was detected on two types of structures:

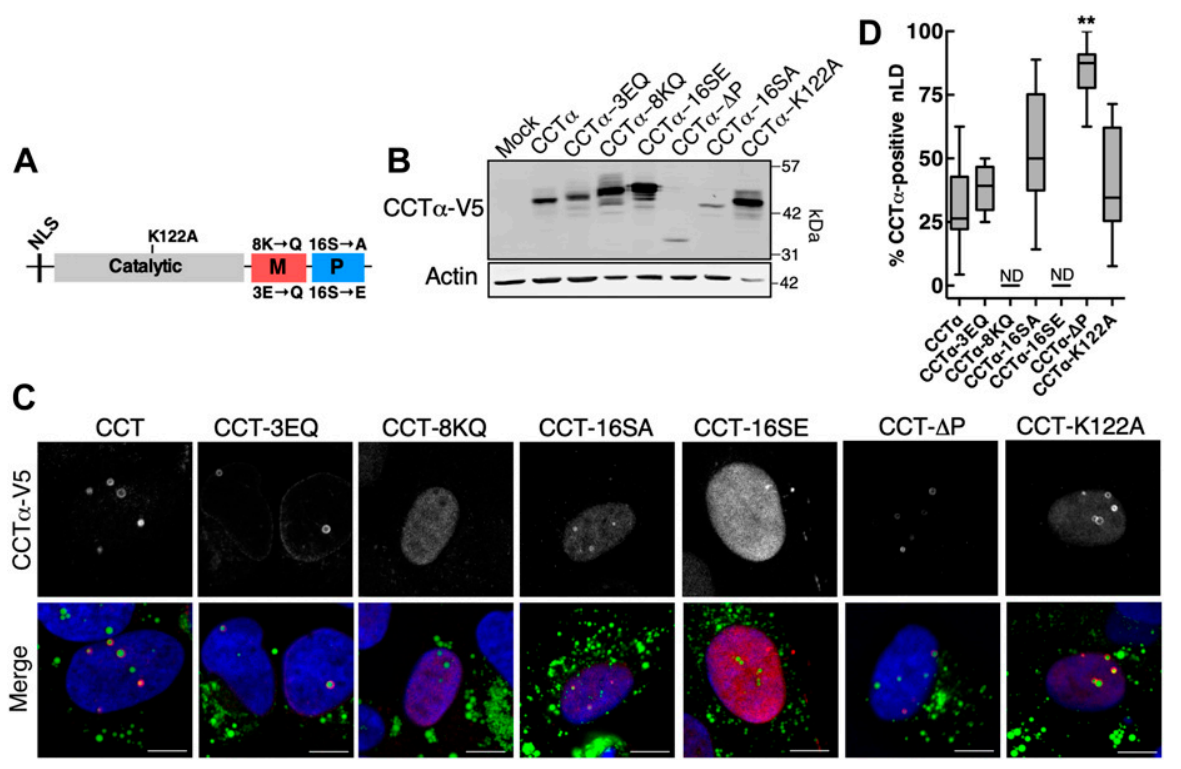

Figure 3. Interaction of CCT $\alpha$ with nuclear lipid droplets (nLDs) is dependent on its $M$ - and P-domains.

(A) Domain organization of CCT $\alpha$ showing the location of catalytic, M-, and P-domain mutations. (B) U2OS cells were transiently transfected with empty vector (Mock) or the indicated CCT $\alpha-\mathrm{V} 5$ constructs and treated with oleate $(400 \mu \mathrm{M})$ for $24 \mathrm{~h}$. Total cell lysates were immunoblotted with $V 5$ and actin antibodies. (B, C) Confocal images of U2OS cells (treated as described in panel B) immunostained with a V5 antibody. LDs and nuclei were visualized with BODIPY 493/503 and propidium iodide, respectively (bar, $10 \mu \mathrm{m}$ ). (D) Quantification of the percentage of nLDs that were positive for wild-type and CCT $\alpha$-V5 mutants presented as box and whisker plots showing the mean and $5^{\text {th }}-95^{\text {th }}$ percentile from analysis of 10-20 fields of cells from 2-3 separate experiments. Significance was determined by one-way ANOVA and Tukey's multiple comparison to $\mathrm{CCT} \alpha$-V5 (ND, not detected; $\left.{ }^{* *} P<0.01\right)$. 


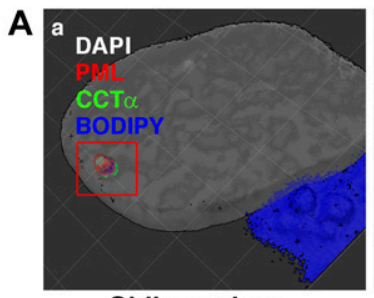

Oblique view

B

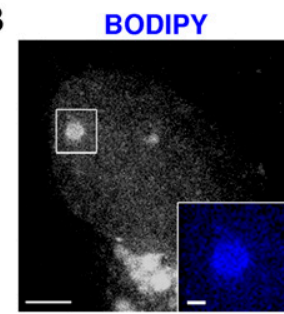

C

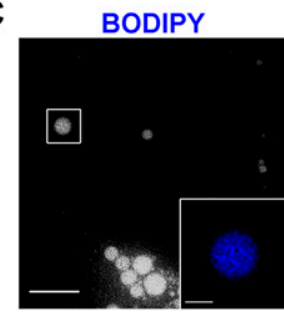

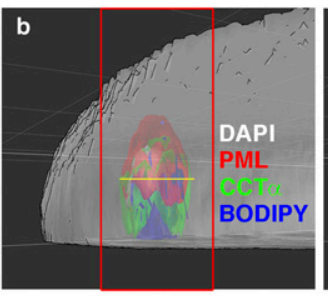

Side View

Widefield Microscopy $\operatorname{cCT} \alpha$
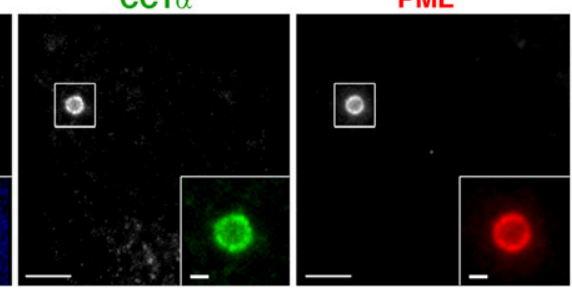

Super Resolution (SRRF)
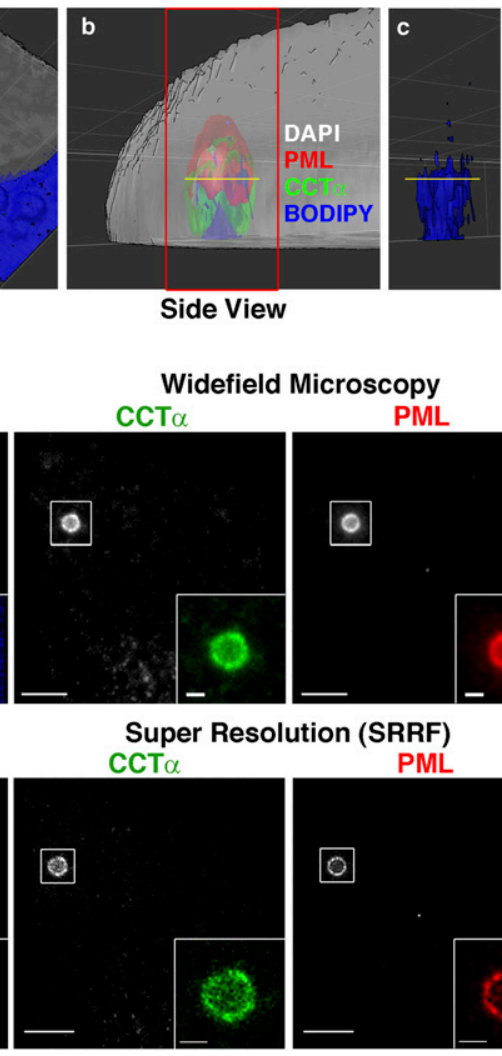

PML

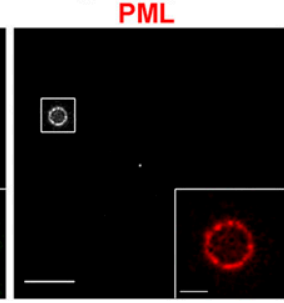

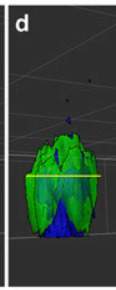
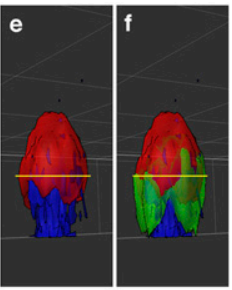

DAPI

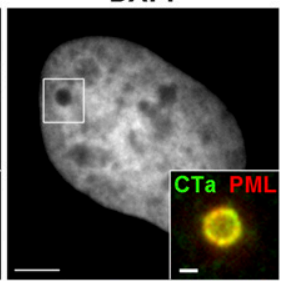

DAPI

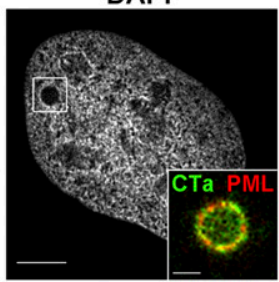

Figure 4. High-resolution imaging of lipid-associated PML structures and CCT $\alpha$ on nuclear lipid droplets (nLDs).

(A) 3D surface reconstruction of image stacks of oleatetreated ( $400 \mu \mathrm{M}$ for $24 \mathrm{~h}$ ) U2OS cells immunostained for PML and CCT $\alpha$. LDs and nuclei were visualized with BODIPY 493/503 and DAPI, respectively. A large nLD was identified in association with CCT $\alpha$ and PML (panel a, red frame). The image was rotated and zoomed to produce a side view of the $\mathrm{nLD}$ (panel $b$, red frame). The framed structure in (b) was assessed by removing the DAPI channel (panels $c-f$ ) to reveal how the $n L D /$ BODIPY (blue), CCT $\alpha$ (green), and PML (red) are associated. (A, B) Wide-field images of the same nLD in (A) imaged at the level of the yellow line indicated in panels $b-f$ (image rotated $~ 90^{\circ}$ clockwise). The nLD is highlighted and magnified in the inset (bar, $5 \mu \mathrm{m}$; inset scale bar, $1 \mu \mathrm{m})$. (B, C) Images of the same field of view in (B) were acquired by Nanol SRRF. The nLD is highlighted and magnified in the inset (bar, $5 \mu \mathrm{m}$; inset bar, $1 \mu \mathrm{m}$ ). small puncta that did not stain with LipidTox Red and PML-positive $n L D s$ (Fig 6A). For the purpose of quantification, we divided LipidTox Red-positive nLDs in U2OS cells into four populations based on the presence or absence of DAG and/or PML (Fig 6B). This revealed that $60 \%$ of total nLDs were negative for both DAG and PML (DAG/PML-). Most of the remaining $n L D s$ contained DAG and PML (79\%), with the remainder containing only DAG (7\%) or PML (14\%) (Fig 6B, insert). The cross-sectional area of DAG/PML- nLDs was significantly reduced compared with total nLDs, whereas the PML+ and DAG/PML+ nLDs were significantly larger (Fig 6C). DAG + nLDs were virtually absent from PML KO cells compared with total and DAG-negative $n L D s$, both of which were also significantly reduced (Fig 6D). We also observed that the nuclear sensor detected DAG on the abundant $\mathrm{nLDs}$ that form in oleate-treated $\mathrm{Cac0} 2$ cells (Fig S8A). Collectively, the results in Figs 5 and 6 indicate that DAG is primarily enriched in large LAPS-positive nLDs, and that loss of these DAG-rich nLDs in PML KO leads to appearance of DAG in CLDs.

To determine whether DAG was a positive effector of CCT $\alpha$ association with nLDs, we determined whether CCT $\alpha$ was preferentially associated with DAG-positive nLDs. Immunofluorescence and line scans through nLDs in oleate-treated U2OS cells showed colocalization between the nuclear DAG sensor and CCTa (Fig 7A). However, in PML KO cells, there was evidence of CCT $\alpha$-positive nLDs that lacked DAG, suggesting poor correlation between DAG content and CCT $\alpha$. This conclusion was supported by quantitation of CCT $\alpha$ and DAG distribution on $\mathrm{nLDs}$. Of the $~ 50 \%$ of $\mathrm{nLDs}$ in U2OS cells that contained DAG and/or CCT $\alpha$, only $43 \%$ contained both DAG and CCTa, suggesting that the DAG content of an nLD is not a strong indicator for recruitment of CCT $\alpha$ (Fig 7B, insert). Quantification of DAG/CCT $\alpha$ distribution on $\mathrm{nLDs}$ in PML KO cells indicated a significant reduction in all four populations compared with control cells, particularly those that contained DAG and DAG/CCT $\alpha$, but the percent distribution of DAG and CCT $\alpha$ on nLDs was similar to control cells (Fig 7B, insert). As well, there was no significant difference in the cross-sectional area of nLDs containing DAG/CCT $\alpha$ in control versus PML KO cells (Fig 7C). The partial (30-40\%) co-localization of CCT $\alpha$ and DAG on nLDs suggests DAG is not a critical factor in CCT $\alpha$ recruitment, whereas electrostatic interactions between lipids and charged residues in the CCT $\alpha \mathrm{M}$-domain could be the driving force for interaction with nLDs (Fig 3).

The source of DAG in nLDs could be Lipin1, a nuclear/cytoplasmic phosphatidic acid (PA) phosphatase that produces DAG for phospholipid and TAG synthesis (Csaki et al, 2013). The human and murine genes encode similar Lipin $1 \alpha$ and $\beta$ splice variants (Peterfy et al, 2005; Croce et al, 2007) that are partially localized to cLDs in COS cells and macrophages (Valdearcos et al, 2011; Wang et al, 2011), potentially recruited by seipin ( $\mathrm{Sim}$ et al, 2012). To determine if Lipin1 recruitment to $\mathrm{nLDs}$ requires LAPS, transiently expressed Lipin $1 \alpha$ and $\beta$ were localized in U2OS and PML KO cells by immunofluorescence microscopy. V5-tagged Lipin1 $\alpha$ was expressed in the nucleus and cytoplasm of untreated U2OS and PML KO cells (Fig 8A). Treatment of U2OS cells with oleate caused extensive association of Lipin1 $\alpha$ with the surface of BODIPY-positive nLDs but not with CLDs (Fig 8B, a-c). Lipin1 $\alpha$ was also localized to the surface of nLDs in Caco2 cells treated with oleate for $24 \mathrm{~h}$ (Fig S8B). To determine the co-localization of Lipin1 $\alpha$ and its product DAG in U2OS 

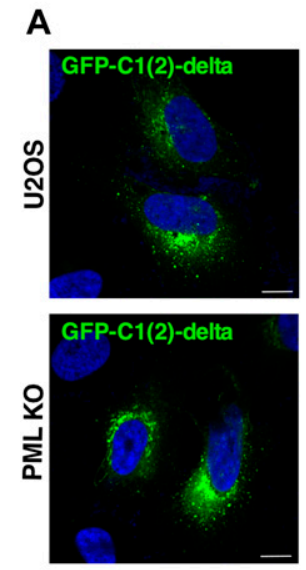

B
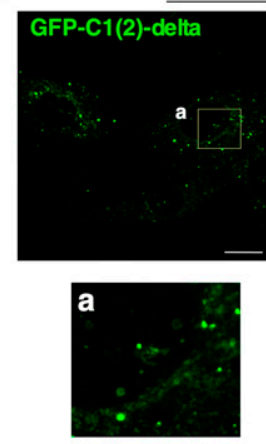

C

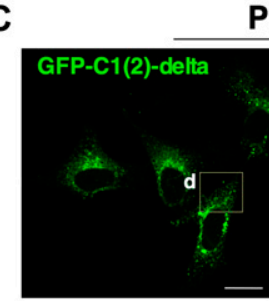

PML KO cells +Oleate

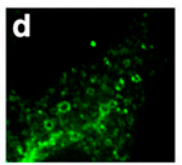

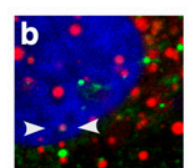
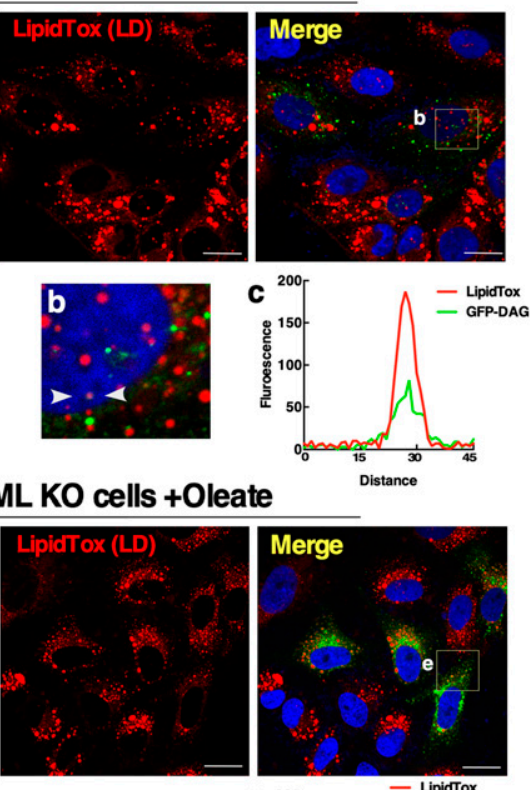

U2OS cells +Oleate
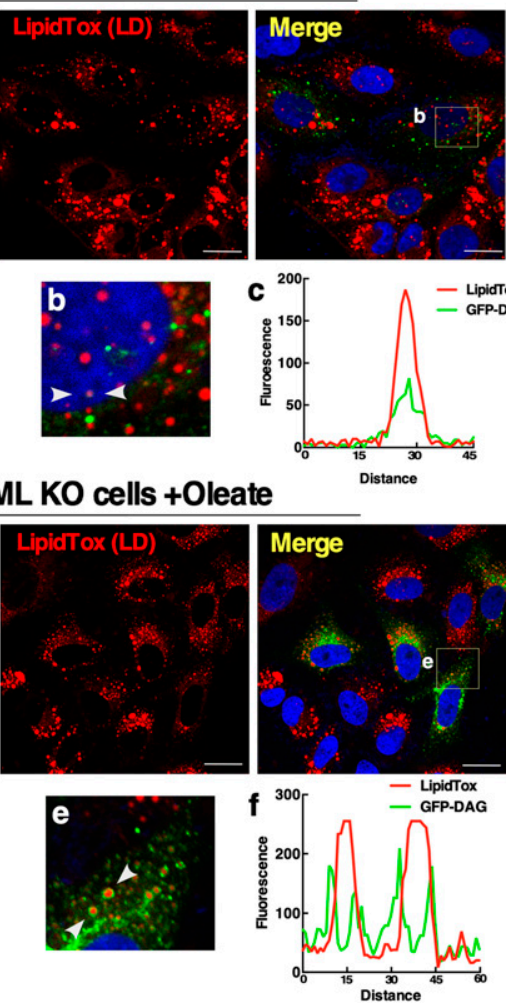

Figure 5. Lipid-associated PML structures control DAG levels on cytoplasmic lipid droplets (cLDs) and nuclear LDs (nLDs).

(A) Localization of the DAG sensor GFP-C1(2) $\delta$ in U2OS and PKL KO cells (bar, $10 \mu \mathrm{m}$ ). (B) U2OS cells expressing GFP-C1(2) $\delta$ were treated with $400 \mu \mathrm{m}$ oleate for $24 \mathrm{~h}$, and LDs were visualized with LipidTox Red (bar, $10 \mu \mathrm{m})$. Magnified areas from GFP and merged images are in panels (a) and (b). Arrows in panel (b) indicate the region selected for an RGB line scan plot (panel c) showing the association of the DAG sensor with a $\mathrm{nLD}$. (A, C) PML KO cells were treated with oleate and stained as described in panel (A) (bar, $10 \mu \mathrm{m}$ ). Magnified areas from GFP and merge images are shown in panels (d) and (e). Arrows in panel (e) indicate the region selected for an RGB line scan plot (panel $f$ ) showing the association of the DAG sensor with CLDs. cells, the distribution and cross-sectional area of Lipin1 $\alpha$ - and DAGcontaining nLDs was quantified. Of the DAG/Lipin1 $\alpha$-positive nLDs, $53 \%$ contained both (Fig 8C, insert), and Lipin1 $\alpha$-positive nLDs were significantly larger than other components of the population (Fig 8D). In oleate-treated PML KO cells, Lipin1 $\alpha$ was diffusely localized in the cytoplasm and nucleus but was virtually absent from nLDs (Fig 8E), a conclusion that was confirmed by quantification of Lipin1 $\alpha$-positive nLDs in PML KO cells compared with controls (Fig $8 F$ ). The Lipin1 $\beta$ isoform also associated with the surface of $n L D s$ but not CLD in oleate-treated U2OS cells (Fig S9A) and was not detected on nLDs in oleate-treated PML KO cells (Fig S9B). The Lipin1 substrate PA was detected with the GFP-nes-2xPABP (Bohdanowicz et al, 2013) biosensor on the plasma membrane and cytoplasmic membranes of control and PML KO cells but was absent from nLDS or CLDs (Fig S10). These data indicate that LAPS are required for association of Lipin1 with nLDs, which could account for the reduced DAG content of nLDs in PML KO cells (Fig 6).

\section{LAPS positively regulate PC and TAG synthesis}

We next tested whether ablating LAPS in PML KO cells and reducing nLD-associated CCT $\alpha$, DAG, and Lipin1 affected cellular lipid synthesis. PC biosynthesis was measured by $\left[{ }^{3} \mathrm{H}\right]$ choline pulse-labeling for 2 and $4 \mathrm{~h}$ in cells treated with or without oleate. PC synthesis in untreated PML KO cells was similar to controls (Fig 9A). However, PC synthesis was poorly activated in oleate-treated PML KO cells and reduced significantly compared with the 2.5 -fold increase caused by oleate in U2OS cells (Fig 9A). PML KO had a minor inhibitory effect on de novo synthesis of fatty acids from $\left[{ }^{3} \mathrm{H}\right]$ acetate that was only significant in cells cultured in lipoprotein-deficient serum (Fig 9B). TAG and CE synthesis in control and PML KO cells was measured by $\left[{ }^{3} \mathrm{H}\right]$ oleate incorporation (Fig $9 \mathrm{C}$ and $\mathrm{D}$ ). Relative to controls, the incorporation of $\left[{ }^{3} \mathrm{H}\right.$ ]oleate into TAG and CE in PML KO cells cultured in FCS or lipoprotein-deficient serum was significantly decreased approximately twofold. To measure the de novo synthesis of TAG, control and PML KO cells were incubated with $100 \mu \mathrm{M}$ oleate for up to $6 \mathrm{~h}$ in the presence of $\left[{ }^{3} \mathrm{H}\right]$ glycerol. In this case, incorporation into TAG in PML KO cells was increased at 1-4 $\mathrm{h}$ but returned to control values at $6 \mathrm{~h}$ (Fig 9E). [ $\left.{ }^{3} \mathrm{H}\right]$ Glycerol incorporation into TAG in U2OS and PML KO cells was similar after oleate treatment for 12 and $24 \mathrm{~h}$ (results not shown). Thus, despite accounting for only $10-15 \%$ of total cellular LDs (Fig 2), nLDs with associated CCT $\alpha$ and Lipin1 activities are important sites for the regulation of $P C$ and TAG synthesis.

\section{Discussion}

The INM is contiguous with the outer nuclear membrane and cytoplasmic ER (Ungricht \& Kutay, 2017) and, thus, could receive lipids by lateral diffusion from the ER (van Meer et al, 2008). However, the INM in yeast (Romanauska \& Kohler, 2018) and mammals (Peterson et al, 2011; Csaki et al, 2013; Aitchison et al, 2015; Haider et al, 2018) harbors lipid biosynthetic enzymes and regulatory proteins suggestive of compartmentalized nuclear lipid synthesis. Analogous to the ER, the INM is the site for de novo biogenesis of nLDs in yeast 
A
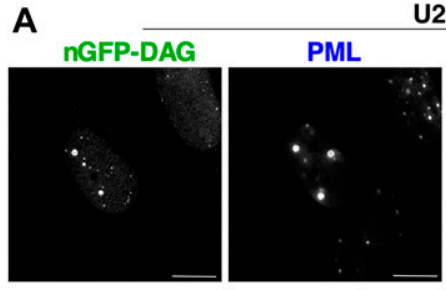

U2OS

B

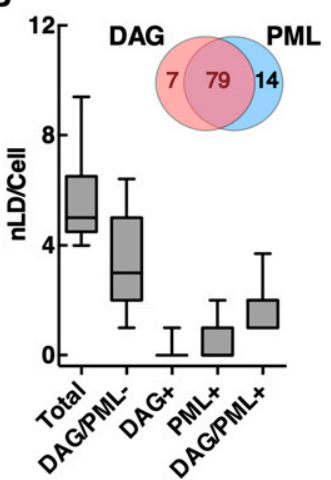

C

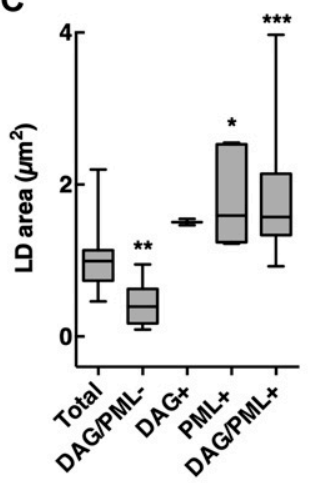

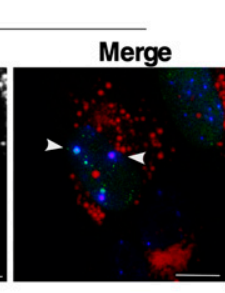

D

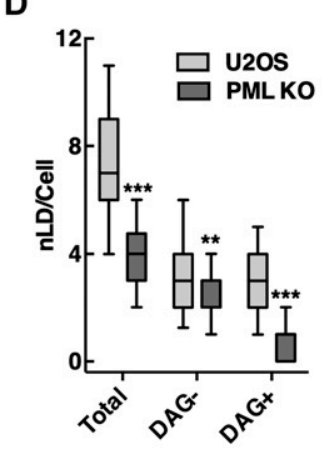

Figure 6. LAP-positive nuclear lipid droplets (nLDs) are enriched in DAG.

(A) U2OS cells transiently expressing nuclear localized GFP-C1(2) $\delta$ (nGFP-DAG) were exposed to oleate $(400 \mu \mathrm{M})$ for $24 \mathrm{~h}$ and were fixed and immunostained with a PML antibody. LDs were visualized with LipidTox Red (bar, $10 \mu \mathrm{m}$ ). Arrows in the merged image indicate the region selected of an RGB line scan plot showing the localization of DAG-GFP with PML and nLDs.

(B) Quantitation of nLDs in oleate-treated U2OS cells containing neither nGFP-DAG or PML (DAG/PML-), nGFP-DAG (DAG+), PML (PML+), or both (DAG/PML+). A Venn diagram shows the percent distribution of nGFPDAG and PML-positive nLDs. (C) The average crosssectional area of nLDs in oleate-treated U2OS cells containing nGFP-DAG and/or PML as described above. (D) Quantification of DAG-negative (-) and DAGnegative (+) nLDs in U2OS and PML KO cells. (B, C, D) Results are presented as box and whisker plots showing the mean and $5^{\text {th }}-95^{\text {th }}$ percentile from analysis of 50-100 cells in three separate experiments. Significance was determined by one-way ANOVA and Tukey's multiple comparison to total nLD area (panel C) or two-tailed $t$ test compared with U2OS controls (panel D) $\left({ }^{\star} P<0.05 ;{ }^{* \star} P<0.01 ;{ }^{* * *} P<0.001\right)$.
(Romanauska \& Kohler, 2018), whereas in hepatoma cells, nLDs are derived from luminal ER LDs that enter the nucleus after dissolution of the INM (Soltysik et al, 2019). PML expression, in particular the PML-II isoform, aids in $\mathrm{nLD}$ biogenesis at the INM by disrupting lamin-free regions of the INM to allow luminal LD release (Ohsaki et al, 2016). Ohsaki et al (2016) also demonstrated that more than $70 \%$ of PML in the nuclei of hepatoma cells were associated with the $n L D s$, but their function and relationship to PML NBs and other PML structures is unknown. We propose that these atypical PML structures be referred to as LAPS to differentiate them from canonical PML NBs and recognize their association with nLDs. LAPS are not essential for nLD formation in U2OS cells but are necessary
A
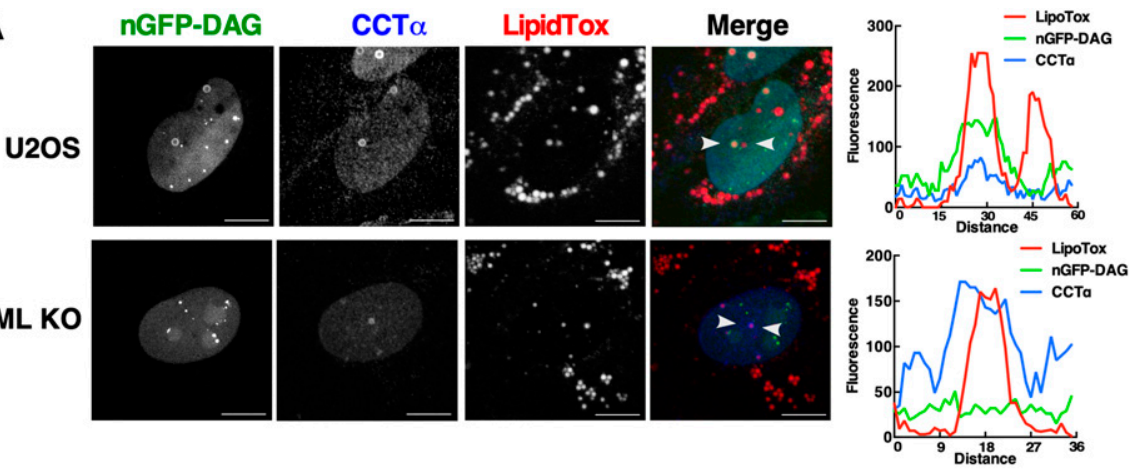

B

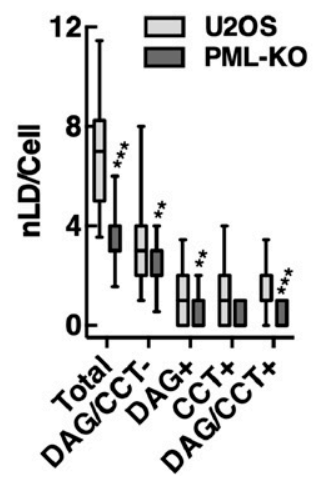

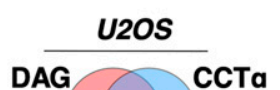

$29 \quad 4328$

PML KO

DAG

$\begin{array}{lll}33 & 31 & 36\end{array}$
C

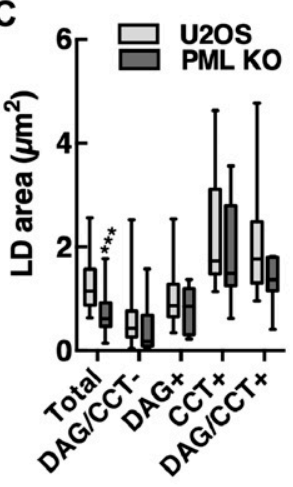

Figure 7. CCT $\alpha$ association with nuclear lipid droplets (nLDs) is DAG independent.

(A) U2OS and PML KO cells transiently expressing a nuclear nGFP-DAG sensor were exposed to $400 \mu \mathrm{M}$ oleate for $24 \mathrm{~h}$, fixed, and immunostained with a $\mathrm{CCT} \alpha$ antibody and incubated with LipidTox Red to visualize LDs (bar, $10 \mu \mathrm{m}$ ). Arrows in the merged image indicate the region selected for an RGB line scan plot showing the association of CCT $\alpha$ and DAG-GFP. (B) Quantification of nLDs in oleate-treated U2OS and PML KO cells containing neither nGFP-DAG or CCT $\alpha$ (CCT $\alpha$ /DAG-GFP-), nGFP-DAG (DAG+), CCT $\alpha$ (CCT+), or both (CCT/DAG+). A Venn diagram shows the percent distribution of nGFP-DAG and CCT $\alpha$-positive nLDs. (c) In oleate-treated U2OS and PML KO cells, the crosssectional area of nLDs containing nGFP-DAG and/or CCT $\alpha$ was measured. (B, C) Results are presented as box and whisker plots showing the mean and $5^{\text {th }}-95^{\text {th }}$ percentile for analysis of 50-100 cells from three separate experiments. Significance was determined by a two-tailed $t$ test compared with matched U2OS controls $\left({ }^{\star *} P<0.01 ;{ }^{* \star *} P<0.001\right)$. 


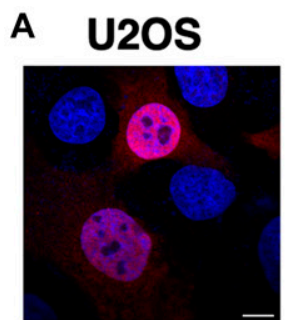

C

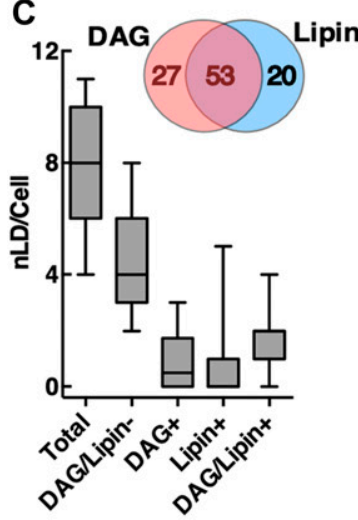

E
PML KO

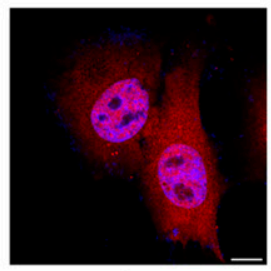

D

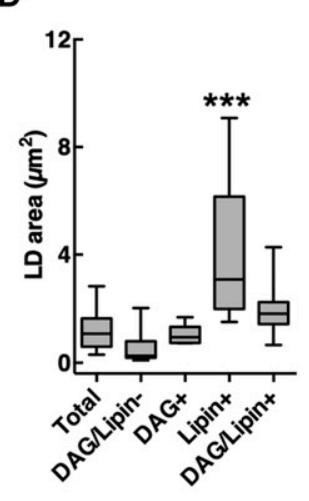

B
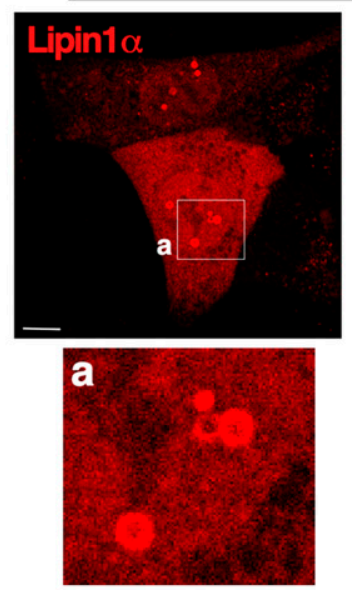

U2OS+oleate
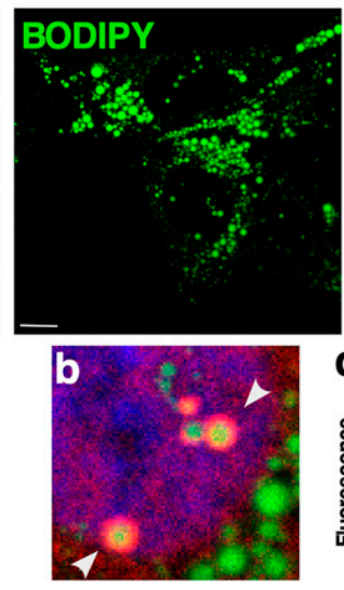
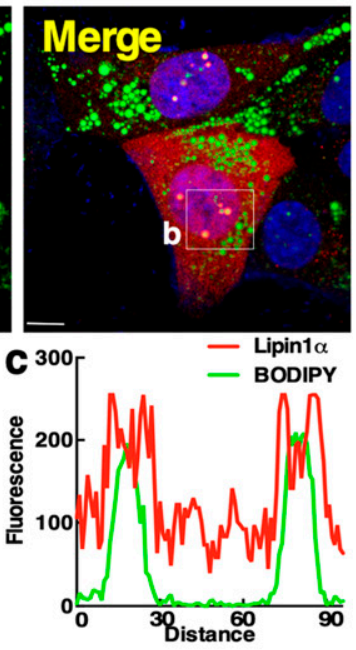

F

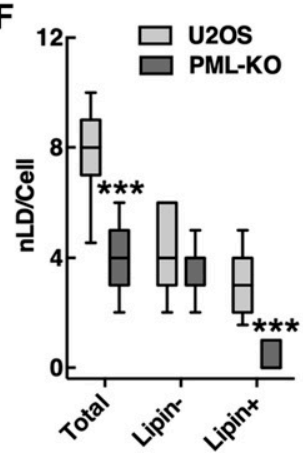

Figure 8. Lipid-associated PML structures promote formation of Lipin1 $\alpha$ with DAG-positive nuclear lipid droplets (nLDs).

(A) U2OS and PML KO cells transiently expressing Lipin $1 \alpha$-V5 were immunostained with a V5 monoclonal antibody, and nuclei were visualized with DAPI (bar, $10 \mu \mathrm{m}$ ). (B) U2OS cells transiently expressing Lipin $1 \alpha$-V5 were treated with $400 \mu \mathrm{M}$ oleate for $24 \mathrm{~h}$ and immunostained with a V5 monoclonal antibody. LDs were visualized with BODIPY 493/503 (bar, $10 \mu \mathrm{m}$ ). Panels (a) and (b) show magnified regions for Lipin1 $\alpha$ and merge images. Arrows show the region in panel (b) selected for an RGB line scan plot (panel c) showing Lipin1 $\alpha$ on the surface of nLDs. (C) Quantification of nLDs in oleate-treated U2OS cells containing neither nGFP-DAG nor Lipin1 $($ Lipin/PML-), nGFP-DAG (DAG+), Lipin1 $\alpha$ (Lipin+), or both (Lipin/DAG+). A Venn diagram shows the percent distribution of nGFP-DAG and Lipin1 $\alpha$-positive nLDs. (D) The cross-sectional area of nLDs containing differing compositions of nGFP-DAG, and Lipin1 $\alpha$ was quantified in oleate-treated U2OS cells. (E) PML KO cells were treated with oleate and immunostained as described above (bar, $10 \mu \mathrm{m}$ ). (F) Quantification of Lipin-negative (-) and Lipin-positive (+) nLDs in U2OS and PML KO cells. (C, D, F) Results are presented as box and whisker plots showing the mean and $5^{\text {th }}-95^{\text {th }}$ percentile for analysis of 50-100 cells from three separate experiments. (C, D, F) Significance was determined by one-way ANOVA and Tukey's multiple comparison (panels C and D) or two-tailed $t$ test compared with control U2OS cells (panel F) (*** $P<0.001$ ).

for the functional maturation of the nLD such that it can recruit CCT $\alpha$ and Lipin1 for the regulation of PC and TAG synthesis.

Coincident with the appearance of LAPS on nLDs in oleatetreated U2OS cells was the loss of canonical PML NBs containing SUM01, DAXX, and SP100. Although oleate treatment caused PML proteins to transfer quantitatively to nLDs without affecting expression, the resultant LAPS were poorly SUMOylated and depleted of PML NB resident proteins. Electron microscopy showed the surface of nLDs had the typical morphology of PML NB (Ohsaki et al, 2016), indicating that LAPS are structurally related but have lost features that are required for PML NB assembly, notably SUMO1 and SP100. Importantly, because LAPS are deficient in these normally constitutive protein components of this nuclear subdomain, by convention and definition, they cannot be called PML NBs. By making this distinction, LAPS are placed within the continuum of PML-containing structures that are already described in the literature with PML NBs at one extreme, followed by LAPS, and at the other extreme, mitotic accumulation of PML proteins (MAPPs) (Dellaire et al, 2006) and PML rods and rosettes in embryonic stem cells (Butler et al, 2009) that are completely devoid of DAXX, SP100, and SUMO1.

PML KO U2OS cells had a 40-50\% reduction in the number of nLDs, a shift to smaller size and reduced association of CCT $\alpha$, Lipin1, and DAG. However, large CCT $\alpha$-positive nLDs in PML KO cells were only reduced by $50 \%$, indicating other mechanisms for nLD biogenesis. Microsomal triglyceride transfer protein inhibitors did not affect nLD formation in U2OS cells suggesting luminal LDs may not be precursors (Soltysik et al, 2019). However, PML-negative nLDs 

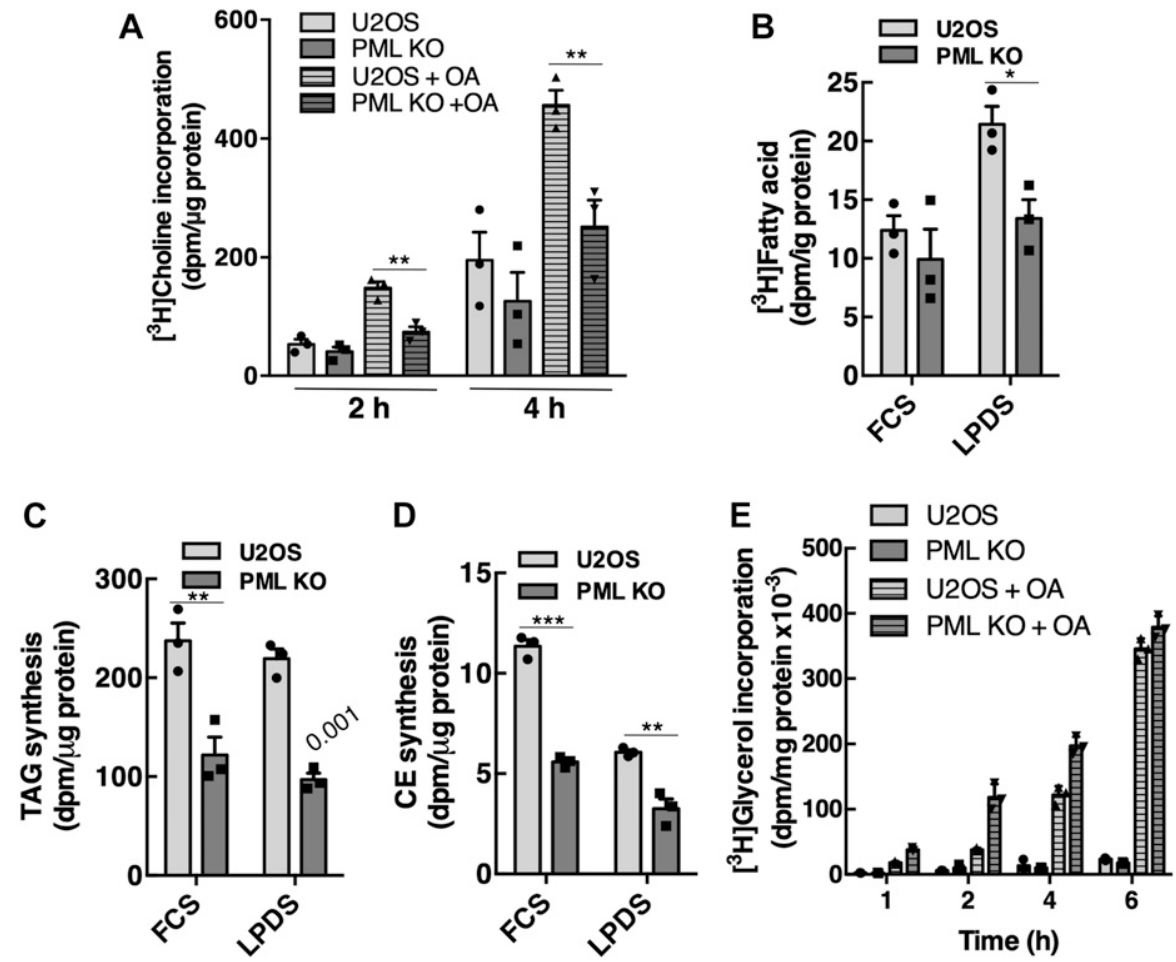

Figure 9. PML KO cells have reduced PC and TAG synthesis.

(A) Cells were cultured for $24 \mathrm{~h}$ in medium containing no addition or $300 \mu \mathrm{M}$ oleate (OA), incubated in cholinefree medium with $\left[{ }^{3} \mathrm{H}\right]$ choline $(1 \mu \mathrm{Ci} / \mathrm{ml})$ for 2 or $4 \mathrm{~h}$, and isotope incorporation into PC was quantified. Results are the mean and SD of five experiments. (B) U2OS and PML KO cells were cultured in FCS or lipoprotein-deficient serum (LPDS) for $24 \mathrm{~h}$, incubated with $\left[{ }^{3} \mathrm{H}\right]$ acetate for $4 \mathrm{~h}$, and incorporation into fatty acids was determined. Results are the mean and SD of three experiments. (C, D) U2OS and PML KO cells were cultured in FCS or LPDS for $18 \mathrm{~h}$ before incubation with $100 \mu \mathrm{M}\left[{ }^{3} \mathrm{H}\right]$ oleate for $4 \mathrm{~h}$ to measure TAG and cholesterol ester (CE) synthesis. Results are the mean and SD of three experiments. (E) U2OS and PML-KO cells were labelled with $\left[{ }^{3} \mathrm{H}\right]$ glycerol $(2 \mu \mathrm{Ci} / \mathrm{ml})$ in the presence or absence of oleate $(\mathrm{OA}, 100 \mu \mathrm{M})$ for up to $\left.6 \mathrm{~h} .{ }^{3} \mathrm{H}\right]$ Glycerol incorporation into TAG is the mean and SD of triplicate determinations from a representative experiment. Statistical comparisons by two-tailed $t$ test $\left({ }^{*} P<0.05 ;{ }^{* *} P<0.01 ;{ }^{* *} P<0.0001\right)$. were observed in close proximity to the NE suggesting that nLDs could originate from ER luminal precursors and/or de novo lipid synthesis at the INM. A clue to the potential mechanisms for nLD biogenesis comes from high-resolution imaging of the polarized localization of LAPS on $\mathrm{nLDS}$. PML-II patches on the INM have been proposed as sites where nLD emerge from the INM (Ohsaki et al, 2016). We used 3D imaging to reveal that these LAPS persist on small and large nLDs and could represent the surface at which the nascent $n L D$ acquired PML proteins as it emerged from the INM. The subsequent maturation of the nLD could be facilitated by the recruitment and activation of CCT $\alpha$ and Lipin1 on exposed surfaces of the LD monolayer, resulting in increased PC and TAG synthesis. The emergence of $\mathrm{nLDs}$ from the INM could also involve the CCT $\alpha$ M-domain, which is capable of inducing positive membrane curvature and expansion of the NR (Lagace \& Ridgway, 2005; Taneva et al, 2012). In this scenario, translocation of CCT $\alpha$ to the INM could induce NR formation and destabilize the bilayer to facilitate the release of nascent nLDs. The presence of large CCT $\alpha$-positive nLDs in PML KO cells supports this concept.

Because PML KO reduced CCT $\alpha$-positive nLDs, we conclude that LAPS are essential to activate PC synthesis in response to prolonged exposure to fatty acids by providing a lipid surface for CCT $\alpha$ activation. Analysis of CCT $\alpha$ mutants showed that association with nLDS was mediated by basic and acidic residues in the $\mathrm{M}$-domain and by dephosphorylation of the P-domain. However, not all 16 phosphosites in the P-domain must be dephosphorylated to promote CCT $\alpha$ association with nLDs. Notably, nLD-associated CCT $\alpha$ is dephosphorylated at S319, whereas Y359 and S362 are constitutively phosphorylated (Yue et al, 2020). To identify lipids that promote CCT $\alpha$ interaction with nLDs, we initially focused on DAG, which induces packing defects in membranes into which the CCT $\alpha$ M-domain can insert (Cornell \& Ridgway, 2015; Cornell, 2016). PML expression was positively correlated with large nLDs that were enriched in DAG. However, DAG and CCT $\alpha$ were not preferentially colocalized in nLDs nor was their relative distribution in $\mathrm{nLDs}$ altered by loss of PML expression. Although the exclusion of CCT $\alpha$ and the biosensor on nLDs could be due to competition for DAG, it is more likely that factors such as surface curvature, enrichment in anionic lipids, or a high $\mathrm{PE} / \mathrm{PC}$ ratio promote the association of CCT $\alpha$. For instance, the high $\mathrm{PE} / \mathrm{PC}$ ratio in insect CLDs relative to human CLDS (Jones et al, 1992; Tauchi-Sato et al, 2002) could be responsible for nuclear export and localization of CCT1 and CCT $\alpha$ on CLDs during oleate loading in Drosophila S2 cells (Krahmer et al, 2011).

The striking PML-dependent redistribution of DAG between nLDs and CLDs was linked to the nuclear PA phosphatase Lipin1 $\alpha$, which co-localized with DAG on nLDs in oleate-treated U2OS and Caco2 cells. Because small nLDs in PML KO cells were relatively devoid of both Lipin1 $\alpha$ and DAG, Lipin1 $\alpha$ appears to provide DAG for nuclear TAG synthesis by diacylglycerol acyltransferase 2, which was localized to nLDs when ectopically expressed in Huh7 cells (Ohsaki et al, 2016). In yeast, nLDs are enriched in PA and DAG as well as enzymes for neutral lipid and phospholipid synthesis (Romanauska \& Kohler, 2018). Interestingly, the yeast Lipin homologue Pah1p was not detected on nLDs but was active on the INM where it produced DAG used for TAG incorporation into nLDs. In U2OS cells, the factor(s) mediating the selective association of Lipin1 with nLDs are uncertain. PA, the Lipin1 substrate that promotes its association with membranes (Ren et al, 2010), was not detected on nLDs or cLDs, suggesting it is below the biosensor detection threshold or the biosensor is not sufficiently PA-specific, as was suggested 
previously (Horchani et al, 2014). Similarly, Lipin1 $\alpha$ and PA were not detected on CLDs in U2OS or PML KO cells, and thus not directly implicated in the accumulation of DAG on CLDS in PML KO cells (Fig 5C).

Metabolic labeling experiments support the concept that LAPS and $n L D s$ are sites for TAG synthesis and regulation. PML KO cells had significantly reduced $\left[{ }^{3} \mathrm{H}\right]$ oleate incorporation into TAG, reduced nLDs, and increased levels of DAG in CLDs, indicative of a block in nuclear and cytoplasmic TAG synthesis. However, PML KO did not inhibit de novo synthesis TAG from $\left[{ }^{3} \mathrm{H}\right]$ glycerol, suggesting a more complex role in oleate uptake and utilization. Prior studies have indicated complex, tissue-specific roles for PML in fatty acid metabolism. For example, PML NB-dependent activation of peroxisome proliferator-activated receptor (PPAR)y co-activator-1 $\alpha$ (PGC- $1 \alpha$ ), PPAR signaling and fatty acid $\beta$-oxidation were shown to provide a growth advantage to breast cancer cells (Carracedo et al, 2012), and controlled asymmetric division and maintenance of hematopoietic stem cells (Ito et al, 2012). In contrast, PML $K O$ mice had tissue-specific enhancement of both fatty acid $\beta$-oxidation and synthesis, increased metabolic rate, and resistance to diet-induced obesity (Cheng et al, 2013). Together with these findings, our results indicate that nLDs and LAPS could have a multi-facetted role in lipid homeostasis involving the recruitment of CCT $\alpha$ and Lipin1 to promote PC and TAG synthesis and storage as well as Lipin1 regulation of a PGC-1 $\alpha /$ PPAR signaling pathways that control fatty acid uptake, storage, and oxidation (Finck et al, 2006).

\section{Materials and Methods}

\section{Cell culture}

U2OS (ATCC HTB-96) and CaCo2 (ATCC HTB-37) cells were cultured in DMEM containing $10 \% \mathrm{FBS}$ (medium $\mathrm{A}$ ) at $37^{\circ} \mathrm{C}$ in a $5 \% \mathrm{CO}_{2}$ atmosphere. CRISPR/Cas9 methodology was used to knockout the expression of all PML isoforms in U2OS cells (Attwood et al, 2019). Unlabeled and $\left[{ }^{3} \mathrm{H}\right]$ oleate/BSA complexes (6:1, mol/mol, $12 \mathrm{mM}$ oleate stock solutions) were prepared as described (Goldstein, 1983).

\section{Plasmid transfection}

Cells were transfected with plasmids encoding murine Lipin1 $\alpha-\mathrm{V} 5$ and Lipin1 $\beta$-V5 (Bou Khalil et al, 2009) (Zemin Yao, University of Ottawa), EGFP-PML isoforms (Bischof et al, 2002) (Oliver Bischof, Institute Pasteur), GFP-C1(2) $\delta$ (Tobias Meyer, plasmid \#21216; Addgene), GFP-nes-2xPABP (Sergio Grinstein, University of Toronto), CCT $\alpha$-16SA and CCT $\alpha$-16SE (P-domain phosphorylation mutants) (Wang \& Kent, 1995), and CCT $\alpha-3 \mathrm{EQ}$ and CCT $\alpha-8 \mathrm{KQ}$ (M-domain mutants) (Johnson et al, 2003; Gehrig et al, 2009) using Lipofectamine $2000(2 \mu \mathrm{l} / \mu \mathrm{g}$ DNA) according to the manufacturer's instructions (Life Technologies). A nuclear DAG sensor (nGFP-DAG) was prepared by subcloning a $\times 2$ NLS cassette into the BsrGI-Afll site of pGFP-C1(2) $\delta$. Experiments were initiated 24-36 h after plasmid transfection.

\section{Immunoblotting}

Cells were lysed in SDS buffer (12.5\% SDS, $30 \mathrm{~mm}$ Tris- $\mathrm{HCl}, 12.5 \%$ glycerol, and $0.01 \%$ bromophenol blue [pH 6.8]) and heated at $95^{\circ} \mathrm{C}$ for $5 \mathrm{~min}$. Lysates were separated by SDS-PAGE, transferred to nitrocellulose membranes, and incubated in TBS $(20 \mathrm{mM}$ Tris- $\mathrm{HCl}$ [pH 7.4] and $500 \mathrm{mM} \mathrm{NaCl):Odyssey} \mathrm{Blocking} \mathrm{Buffer} \mathrm{(5:1,} \mathrm{vol/vol)} \mathrm{for}$ $1 \mathrm{~h}$. Nitrocellulose was blotted using primary antibodies against human CCT $\alpha$ (Morton et al, 2013), PML (rabbit polyclonal A301-167A; Bethyl Laboratories), V5 monoclonal (MCA-1360; Bio-Rad), or $\beta$-actin (mouse monoclonal AC15; Sigma-Aldrich). Proteins were visualized with goat antimouse or goat antirabbit IRDye-800 or IRDye-680 secondary antibodies (LI-COR Biosciences) using an Odyssey Imaging System and application software (v3.0).

\section{Analysis of PC synthesis using $\left[{ }^{3} \mathrm{H}\right]$ choline incorporation}

U2OS and PML KO cells were incubated in the presence or absence of $400 \mu \mathrm{M}$ oleate/BSA for $24 \mathrm{~h}$. Cells were rinsed twice with cholinefree medium $A$ and then incubated with choline-free medium $A$ containing $\left[{ }^{3} \mathrm{H}\right]$ choline $(2 \mu \mathrm{Ci} / \mathrm{ml})$ for 2 and $4 \mathrm{~h}$. After isotope labeling, the cells were rinsed with cold PBS, harvested in methanol: water (5:4, vol/vol), $\left[{ }^{3} \mathrm{H}\right] \mathrm{PC}$ was extracted in chloroform/methanol, and the radioactivity quantified by liquid scintillation counting and normalized to total cellular protein (Storey et al, 1997).

\section{Analysis of fatty acid and TAG synthesis}

U2OS and PML KO cells were incubated with $2.5 \mu \mathrm{Ci} / \mathrm{ml}\left[{ }^{3} \mathrm{H}\right]$ acetate for $4 \mathrm{~h}$ to measure fatty acid synthesis (Brown et al, 1978). Briefly, lipid extracts from cells were saponified in ethanol and 50\% potassium hydroxide (wt/vol) for $1 \mathrm{~h}$ at $60^{\circ} \mathrm{C}$ and extracted with hexane. The hydrolysate was then acidified with $\mathrm{HCl}(\mathrm{pH}<3)$, and radiolabeled fatty acids were extracted with hexane and quantified by liquid scintillation counting. Incorporation of $\left[{ }^{3} \mathrm{H}\right]$ acetate into fatty acids was normalized to total cellular protein.

TAG and CE synthesis was determined by incubating cells with $100 \mu \mathrm{M}\left[{ }^{3} \mathrm{H}\right.$ ]oleate/BSA. After $4 \mathrm{~h}$, the cells were rinsed twice with cold $150 \mathrm{mM} \mathrm{NaCl}$ and $50 \mathrm{mM}$ Tris- $\mathrm{HCl}$ (pH 7.4) with $2 \mathrm{mg} / \mathrm{ml} \mathrm{BSA}$ and once with the same buffer without BSA. $\left[{ }^{3} \mathrm{H}\right]$ Oleate-labeled lipids were extracted from dishes with hexane:isopropanol (3:2, vol/vol). Samples were dried under nitrogen, resolved by thin-layer chromatography in hexane:diethyl ether:acetic acid (90:30:1, vol/vol), and radioactivity in $\left[{ }^{3} \mathrm{H}\right] \mathrm{TAG}$ and $\left[{ }^{3} \mathrm{H}\right] \mathrm{CE}$ was quantified by scintillation counting and normalized to total cellular protein. De novo TAG synthesis was determined by incubating cells with or without $400 \mu \mathrm{M}$ oleate/BSA in the presence of $2 \mu \mathrm{Ci} / \mathrm{ml}\left[{ }^{3} \mathrm{H}\right]$ glycerol. The cells were rinsed with cold PBS, and radioactive lipids were extracted and quantified as described above.

\section{Immunofluorescence microscopy}

Cells cultured on glass coverslips were fixed with $4 \%$ (wt/vol) paraformaldehyde and permeabilized for $10 \mathrm{~min}$ with $0.1 \%$ (wt) vol) Triton X-100 at room temperature. Coverslips were incubated with primary antibodies against CCT $\alpha, \mathrm{PML}$ (monoclonal E-11; Santa Cruz), PML (rabbit polyclonal A301-167A; Bethyl Laboratories), 
LMNA/C (monoclonal 4C11; Cell Signaling), emerin (polyclonal FL254; Santa Cruz), DAXX (polyclonal D7810; Sigma-Aldrich), SP100 (polyclonal PA5-53602; Invitrogen), SUMO1 (rabbit monoclonal Y299, Ab32058; Abcam), or V5 in PBS containing 1\% (wt/vol) BSA. This was followed by secondary AlexFluor488-, 594-, and 647-conjugated goat antirabbit or antimouse secondary antibodies (Thermo Fisher Scientific). To visualize nuclear and CLDS, BODIPY 493/503 or LipidTox Red was diluted 1:1,000 or 1:500, respectively, and incubated with the secondary antibodies. Coverslips were mounted on glass slides in Mowiol 4-88, and confocal imaging was performed using a Zeiss LSM510 or LSM710 laser scanning confocal microscope with a Plan-Apochromat 100× (1.4 NA) oil immersion objective. LD cross-sectional area in confocal images was quantified using ImageJ software (v1.47, National Institutes of Health). Images were converted to 8-bit, the threshold was adjusted, and the "analyze particle" command was used to exclude on edges. The percent distribution of LDs within a binned area group was quantified.

Quantification of SP100, SUMO1, and DAXX co-localization with PML on BODIPY-positive nLDs was binned into strong, weak, and none association groupings. Strong association was indicated by co-localization of SP100, DAXX, and SUMO1 throughout the PML structure with a signal intensity that was $>50 \%$ of that for PML. Weak association was scored as partial co-localized with PML on nLDs and a signal intensity $<50 \%$ relative to PML. None-association indicated no signal associated with PML-positive nLDs.

For SRRF imaging (Gustafsson et al, 2016), immunostained cells (described above) were observed using a $100 \times$ Plan-Apochromat (1.46 NA) oil immersion objective lens (Zeiss) by wide-field imaging on a Marianis microscope (Intelligent Imaging Innovations, 3i) based on a Zeiss Axio Cell Observer equipped with LED-based illumination via a SPECTRA III Light Engine (Lumencor), and a Prime BSI back-illuminated scientific complementary metal-oxidesemiconductor (sCMOS) camera (Teledyne Photometrics). This imaging configuration resulted in an effective pixel size of $65 \times 65$ $\mathrm{nm}$ in the captured images. To generate super-resolution images, 100 wide-field images were captured at $10 \mathrm{~ms} /$ frame using SlideBook 6 software and then exported to Image (version 1.52, NIH) in a 16-bit Open Microscopy Environment-Tagged Image File Format. Image processing used a custom SRRF algorithm (NanoJ-LiveSRRF, available on request from Ricardo Henriques, University College London/Francis Crick Institute, UK) with the following settings: radius 3 , sensitivity 2 , magnification 4 , average temporal analysis, and with intensity weighting and both vibration and macro-pixel correction turned on. NanoJ-LiveSRRF is the newest implementation of NanoJ-SRRF within the ImageJ software, available upon request as above. However, NanoJ-SRRF is already freely available (Gustafsson et al, 2016).

For confocal imaging and 3D volume rendering, the cells were immunostained as above and imaged using a $100 \mathrm{X}$ PlanApochromat (1.46 NA) oil immersion objective lens (Zeiss) by spinning-disk confocal microscopy on a Marianis microscope (Intelligent Imaging Innovations, 3i) based on a Zeiss Axio Cell Observer equipped with Yokagawa CSU-X1 spinning-disk unit, four laser lines (405, 488, 560 and $640 \mathrm{~nm}$ ), and a Prime95B (Teledyne/ Photometrics). 3D volume rendering of confocal image stacks was performed using SlideBook 6 software (3i).

\section{Supplementary Information}

Supplementary Information is available at https://doi.org/10.26508/lsa 202000751.

\section{Acknowledgements}

We would like to thank Romain Laine and Ricardo Henriques (University College London/Francis Crick Institute, UK) for providing guidance on SRRF imaging and optimization. This work was funded by a Project Grant to $G$ Dellaire and ND Ridgway from the Canadian Institutes of Health Research (PJT62390) and the Bernard and Winnifred Lockwood Endowment for Research. I Lee was supported by a scholarship from the Nova Scotia Health Research Foundation. G Dellaire and ND Ridgway are Senior Scientists of the Beatrice Hunter Cancer Research Institute.

\section{Author Contributions}

J Lee: formal analysis, investigation, methodology, and writing-original draft, review, and editing.

J Salsman: formal analysis, investigation, methodology, and writing-original draft, review, and editing.

J Foster: formal analysis, investigation, methodology, and writing-review and editing.

G Dellaire: conceptualization, data curation, supervision, funding acquisition, methodology, project administration, and writing-original draft, review, and editing.

ND Ridgway: conceptualization, data curation, supervision, funding acquisition, methodology, project administration, and writing-original draft, review, and editing.

\section{Conflict of Interest Statement}

The authors declare that they have no conflict of interest.

\section{References}

Aitchison AJ, Arsenault DJ, Ridgway ND (2015) Nuclear-localized CTP: phosphocholine cytidylyltransferase alpha regulates phosphatidylcholine synthesis required for lipid droplet biogenesis. Mol Biol Cell 26: 2927-2938. doi:10.1091/mbc.e15-03-0159

Arnold RS, Cornell RB (1996) Lipid regulation of CTP: Phosphocholine cytidylyltransferase: Electrostatic, hydrophobic, and synergistic interactions of anionic phospholipids and diacylglycerol. Biochemistry 35: 9917-9924. doi:10.1021/bi960397c

Arnold RS, DePaoli-Roach AA, Cornell RB (1997) Binding of CTP: phosphocholine cytidylyltransferase to lipid vesicles: Diacylglycerol and enzyme dephosphorylation increase the affinity for negatively charged membranes. Biochemistry 36: 6149-6156. doi:10.1021/ bi970023z

Attwood KM, Salsman J, Chung D, Mathavarajah S, Van Iderstine C, Dellaire G (2019) PML isoform expression and DNA break location relative to PML nuclear bodies impacts the efficiency of homologous recombination. Biochem Cell Biol: 1-13. doi:10.1139/bcb-2019-0115

Bartz R, Li WH, Venables B, Zehmer JK, Roth MR, Welti R, Anderson RG, Liu P, Chapman KD (2007) Lipidomics reveals that adiposomes store ether lipids and mediate phospholipid traffic. J Lipid Res 48: 837-847. doi:10.1194/jlr.m600413-jlr200 
Bischof O, Kirsh O, Pearson M, Itahana K, Pelicci PG, Dejean A (2002) Deconstructing PML-induced premature senescence. EMBO J 21: 3358-3369. doi:10.1093/emboj/cdf341

Bohdanowicz M, Schlam D, Hermansson M, Rizzuti D, Fairn GD, Ueyama T, Somerharju P, Du G, Grinstein S (2013) Phosphatidic acid is required for the constitutive ruffling and macropinocytosis of phagocytes. Mol Biol Cell 24: 1700-1712, S1-S7. doi:10.1091/mbc.e12-11-0789

Bou Khalil M, Sundaram M, Zhang HY, Links PH, Raven JF, Manmontri B, Sariahmetoglu M, Tran K, Reue K, Brindley DN, et al (2009) The level and compartmentalization of phosphatidate phosphatase-1 (lipin-1) control the assembly and secretion of hepatic VLDL. J Lipid Res 50 : 47-58. doi:10.1194/jlr.m800204-jlr200

Brown MS, Faust JR, Goldstein JL (1978) Induction of 3-hydroxy-3methylglutaryl coenzyme A reductase activity in human fibroblasts incubated with compactin (ML-236B), a competitive inhibitor of the reductase. J Biol Chem 253: 1121-1128.

Butler JT, Hall LL, Smith KP, Lawrence JB (2009) Changing nuclear landscape and unique PML structures during early epigenetic transitions of human embryonic stem cells. J Cell Biochem 107: 609-621. doi:10.1002/ jcb.22183

Carracedo A, Weiss D, Leliaert AK, Bhasin M, de Boer VC, Laurent G, Adams AC, Sundvall M, Song SJ, Ito K, et al (2012) A metabolic prosurvival role for PML in breast cancer. J Clin Invest 122: 3088-3100. doi:10.1172/jci62129

Cheng X, Guo S, Liu Y, Chu H, Hakimi P, Berger NA, Hanson RW, Kao HY (2013) Ablation of promyelocytic leukemia protein (PML) re-patterns energy balance and protects mice from obesity induced by a Western diet. J Biol Chem 288: 29746-29759. doi:10.1074/jbc.m113.487595

Ching RW, Dellaire G, Eskiw CH, Bazett-Jones DP (2005) PML bodies: A meeting place for genomic loci? J Cell Sci 118: 847-854. doi:10.1242/jcs.01700

Codazzi F, Teruel MN, Meyer T (2001) Control of astrocyte Ca(2+) oscillations and waves by oscillating translocation and activation of protein kinase C. Curr Biol 11: 1089-1097. doi:10.1016/s0960-9822(01)00326-8

Cornell RB (2016) Membrane lipid compositional sensing by the inducible amphipathic helix of CCT. Biochim Biophys Acta 1861: 847-861. doi:10.1016/j.bbalip.2015.12.022

Cornell RB, Ridgway ND (2015) CTP:phosphocholine cytidylyltransferase: Function, regulation, and structure of an amphitropic enzyme required for membrane biogenesis. Prog Lipid Res 59: 147-171. doi:10.1016/j.plipres.2015.07.001

Croce MA, Eagon JC, LaRiviere LL, Korenblat KM, Klein S, Finck BN (2007) Hepatic lipin 1beta expression is diminished in insulin-resistant obese subjects and is reactivated by marked weight loss. Diabetes 56: 2395-2399. doi:10.2337/db07-0480

Csaki LS, Dwyer JR, Fong LG, Tontonoz P, Young SG, Reue K (2013) Lipins, lipinopathies, and the modulation of cellular lipid storage and signaling. Prog Lipid Res 52: 305-316. doi:10.1016/j.plipres.2013.04.001

Culley S, Albrecht D, Jacobs C, Pereira PM, Leterrier C, Mercer J, Henriques R (2018) Quantitative mapping and minimization of super-resolution optical imaging artifacts. Nat Methods 15: 263-266. doi:10.1038/ nmeth. 4605

Dellaire G, Bazett-Jones DP (2004) PML nuclear bodies: Dynamic sensors of DNA damage and cellular stress. Bioessays 26: 963-977. doi:10.1002/ bies.20089

Dellaire G, Eskiw CH, Dehghani H, Ching RW, Bazett-Jones DP (2006) Mitotic accumulations of PML protein contribute to the re-establishment of PML nuclear bodies in G1. J Cell Sci 119: 1034-1042. doi:10.1242/jcs.02817

Finck BN, Gropler MC, Chen Z, Leone TC, Croce MA, Harris TE, Lawrence JC, Jr, Kelly DP (2006) Lipin 1 is an inducible amplifier of the hepatic PGC1alpha/PPARalpha regulatory pathway. Cell Metab 4: 199-210. doi:10.1016/j.cmet.2006.08.005

Gehrig K, Morton CC, Ridgway ND (2009) Nuclear export of the rate-limiting enzyme in phosphatidylcholine synthesis is mediated by its membrane binding domain. J Lipid Res 50: 966-976. doi:10.1194/ jlr.m800632-jlr200

Goldstein JL, Basu SK, Brown MS (1983) Receptor mediated endocytosis of low-density lipoprotein in cultured cells. Methods Enzymol 98: 241-260. doi:10.1016/0076-6879(83)98152-1

Guo Y, Walther TC, Rao M, Stuurman N, Goshima G, Terayama K, Wong JS, Vale RD, Walter P, Farese RV (2008) Functional genomic screen reveals genes involved in lipid-droplet formation and utilization. Nature 453: 657-661. doi:10.1038/nature06928

Gustafsson N, Culley S, Ashdown G, Owen DM, Pereira PM, Henriques R (2016) Fast live-cell conventional fluorophore nanoscopy with ImageJ through super-resolution radial fluctuations. Nat Commun 7: 12471. doi:10.1038/ncomms12471

Haider A, Wei YC, Lim K, Barbosa AD, Liu CH, Weber U, Mlodzik M, Oras K, Collier S, Hussain MM, et al (2018) PCYT1A regulates phosphatidylcholine homeostasis from the inner nuclear membrane in response to membrane stored curvature elastic stress. Dev Cell 45: 481-495.e8. doi:10.1016/j.devcel.2018.04.012

Henne WM, Reese ML, Goodman JM (2018) The assembly of lipid droplets and their roles in challenged cells. EMBO J 37: e98947. doi:10.15252/ embj.201898947

Horchani H, de Saint-Jean M, Barelli H, Antonny B (2014) Interaction of the Spo20 membrane-sensor motif with phosphatidic acid and other anionic lipids, and influence of the membrane environment. PLOS One 9: e113484. doi:10.1371/journal.pone.0113484

Ishov AM, Sotnikov AG, Negorev D, Vladimirova OV, Neff N, Kamitani T, Yeh ET, Strauss JF 3rd, Maul GG (1999) PML is critical for ND10 formation and recruits the PML-interacting protein daxx to this nuclear structure when modified by SUMO-1. J Cell Biol 147: 221-234. doi:10.1083/ jcb.147.2.221

Ito K, Carracedo A, Weiss D, Arai F, Ala U, Avigan DE, Schafer ZT, Evans RM, Suda T, Lee CH, et al (2012) A PML-PPAR-delta pathway for fatty acid oxidation regulates hematopoietic stem cell maintenance. Nat Med 18: 1350-1358. doi:10.1038/nm.2882

Johnson JE, Xie M, Singh LM, Edge R, Cornell RB (2003) Both acidic and basic amino acids in an amphitropic enzyme, CTP:phosphocholine cytidylyltransferase, dictate its selectivity for anionic membranes. J Biol Chem 278: 514-522. doi:10.1074/jbc.m206072200

Jones HE, Harwood JL, Bowen ID, Griffiths G (1992) Lipid composition of subcellular membranes from larvae and prepupae of Drosophila melanogaster. Lipids 27: 984-987. doi:10.1007/bf02535576

Krahmer N, Guo Y, Wilfling F, Hilger M, Lingrell S, Heger K, Newman HW, Schmidt-Supprian M, Vance DE, Mann M, et al (2011) Phosphatidylcholine synthesis for lipid droplet expansion is mediated by localized activation of CTP:phosphocholine cytidylyltransferase. Cell Metab 14: 504-515. doi:10.1016/ j.cmet.2011.07.013

Lagace TA, Ridgway ND (2005) The rate-limiting enzyme in phosphatidylcholine synthesis regulates proliferation of the nucleoplasmic reticulum. Mol Biol Cell 16: 1120-1130. doi:10.1091/ mbc.e04-10-0874

Lagrutta LC, Montero-Villegas S, Layerenza JP, Sisti MS, Garcia de Bravo MM, Ves-Losada A (2017) Reversible nuclear-lipid-droplet morphology induced by oleic acid: A link to cellular-lipid metabolism. PLoS One 12: e0170608. doi:10.1371/journal.pone.0170608

Morton CC, Aitchison AJ, Gehrig K, Ridgway ND (2013) A mechanism for suppression of the CDP-choline pathway during apoptosis. J Lipid Res 54: 3373-3384. doi:10.1194/jlr.m041434

Northwood IC, Tong AH, Crawford B, Drobnies AE, Cornell RB (1999) Shuttling of CTP: Phosphocholine cytidylyltransferase between the nucleus and endoplasmic reticulum accompanies the wave of phosphatidylcholine synthesis during the $\mathrm{G}(0) \rightarrow \mathrm{G}(1)$ transition. J Biol Chem 274: 26240-26248. doi:10.1074/jbc.274.37.26240 
Ohsaki Y, Kawai T, Yoshikawa Y, Cheng J, Jokitalo E, Fujimoto T (2016) PML isoform II plays a critical role in nuclear lipid droplet formation. J Cell Biol 212: 29-38. doi:10.1083/jcb.201507122

Payne F, Lim K, Girousse A, Brown RJ, Kory N, Robbins A, Xue Y, Sleigh A, Cochran E, Adams C, et al (2014) Mutations disrupting the Kennedy phosphatidylcholine pathway in humans with congenital lipodystrophy and fatty liver disease. Proc Natl Acad Sci U S A 111: 8901-8906. doi:10.1073/pnas.1408523111

Peterfy M, Phan J, Reue K (2005) Alternatively spliced lipin isoforms exhibit distinct expression pattern, subcellular localization, and role in adipogenesis. J Biol Chem 280: 32883-32889. doi:10.1074/ jbc.m503885200

Peterson TR, Sengupta SS, Harris TE, Carmack AE, Kang SA, Balderas E, Guertin DA, Madden KL, Carpenter AE, Finck BN, et al (2011) mTOR complex 1 regulates lipin 1 localization to control the SREBP pathway. Cell 146: 408-420. doi:10.1016/j.cell.2011.06.034

Prevost C, Sharp ME, Kory N, Lin Q, Voth GA, Farese RV, Jr, Walther TC (2018) Mechanism and determinants of amphipathic helix-containing protein targeting to lipid droplets. Dev Cell 44: 73-86.e4. doi:10.1016/ j.devcel.2017.12.011

Ren H, Federico L, Huang H, Sunkara M, Drennan T, Frohman MA, Smyth SS, Morris AJ (2010) A phosphatidic acid binding/nuclear localization motif determines lipin1 function in lipid metabolism and adipogenesis. Mol Biol Cell 21: 3171-3181. doi:10.1091/mbc.e10-01-0073

Romanauska A, Kohler A (2018) The inner nuclear membrane is a metabolically active territory that generates nuclear lipid droplets. Cell 174: 700-715. doi:10.1016/j.cell.2018.05.047

Sim MF, Dennis RJ, Aubry EM, Ramanathan N, Sembongi H, Saudek V, Ito D, O'Rahilly S, Siniossoglou S, Rochford JJ (2012) The human lipodystrophy protein seipin is an ER membrane adaptor for the adipogenic PA phosphatase lipin 1. Mol Metab 2: 38-46. doi:10.1016/ j.molmet.2012.11.002

Soltysik K, Ohsaki Y, Tatematsu T, Cheng J, Fujimoto T (2019) Nuclear lipid droplets derive from a lipoprotein precursor and regulate phosphatidylcholine synthesis. Nat Commun 10: 473. doi:10.1038/ s41467-019-08411-x

Sternsdorf T, Jensen K, Will H (1997) Evidence for covalent modification of the nuclear dot-associated proteins PML and Sp100 by PIC1/SUMO-1. I Cell Biol 139: 1621-1634. doi:10.1083/jcb.139.7.1621

Storey MK, Byers DM, Cook HW, Ridgway ND (1997) Decreased phosphatidylcholine biosynthesis and abnormal distribution of CTP: phosphocholine cytidylyltransferase in cholesterol auxotrophic Chinese hamster ovary cells. J Lipid Res 38: 711-722.

Sztalryd C, Brasaemle DL (2017) The perilipin family of lipid droplet proteins: Gatekeepers of intracellular lipolysis. Biochim Biophys Acta Mol Cell Biol Lipids 1862: 1221-1232. doi:10.1016/j.bbalip.2017.07.009

Taneva SG, Lee JM, Cornell RB (2012) The amphipathic helix of an enzyme that regulates phosphatidylcholine synthesis remodels membranes into highly curved nanotubules. Biochim Biophys Acta 1818: 1173-1186. doi:10.1016/j.bbamem.2012.01.006
Tauchi-Sato K, Ozeki S, Houjou T, Taguchi R, Fujimoto T (2002) The surface of lipid droplets is a phospholipid monolayer with a unique fatty acid composition. J Biol Chem 277: 44507-44512. doi:10.1074/jbc.m207712200

Ungricht R, Kutay U (2017) Mechanisms and functions of nuclear envelope remodelling. Nat Rev Mol Cell Biol 18: 229-245. doi:10.1038/ nrm.2016.153

Uzbekov R, Roingeard P (2013) Nuclear lipid droplets identified by electron microscopy of serial sections. BMC Res Notes 6: 386. doi:10.1186/17560500-6-386

Valdearcos M, Esquinas E, Meana C, Gil-de-Gomez L, Guijas C, Balsinde J, Balboa MA (2011) Subcellular localization and role of lipin-1 in human macrophages. J Immunol 186: 6004-6013. doi:10.4049/ jimmunol.1003279

Van Damme E, Laukens K, Dang TH, Van Ostade X (2010) A manually curated network of the PML nuclear body interactome reveals an important role for PML-NBs in SUMOylation dynamics. Int J Biol Sci 6: 51-67. doi:10.7150/ijbs.6.51

van Meer G, Voelker DR, Feigenson GW (2008) Membrane lipids: Where they are and how they behave. Nat Rev Mol Cell Biol 9: 112-124. doi:10.1038/ nrm2330

Walther TC, Farese RV, Jr (2012) Lipid droplets and cellular lipid metabolism. Annu Rev Biochem 81: 687-714. doi:10.1146/annurev-biochem-061009102430

Wang H, Zhang J, Qiu W, Han GS, Carman GM, Adeli K (2011) Lipin-1gamma isoform is a novel lipid droplet-associated protein highly expressed in the brain. FEBS Lett 585: 1979-1984. doi:10.1016/j.febslet.2011.05.035

Wang Y, Kent C (1995) Effects of altered phosphorylation sites on the properties of CTP:phosphocholine cytidylyltransferase. I Biol Chem 270: 17843-17849. doi:10.1074/jbc.270.30.17843

Xie M, Smith JL, Ding Z, Zhang D, Cornell RB (2004) Membrane binding modulates the quaternary structure of CTP:phosphocholine cytidylyltransferase. J Biol Chem 279: 28817-28825. doi:10.1074/ jbc.m403311200

Yue L, McPhee MJ, Gonzalez K, Charman M, Lee J, Thompson J, Winkler DFH, Cornell RB, Pelech S, Ridgway ND (2020) Differential dephosphorylation of CTP:phosphocholine cytidylyltransferase upon translocation to nuclear membranes and lipid droplets. Mol Biol Cell 31: 1047-1059. doi:10.1091/mbc.E20-01-0014

Zhong S, Muller S, Ronchetti S, Freemont PS, Dejean A, Pandolfi PP (2000) Role of SUMO-1-modified PML in nuclear body formation. Blood 95: 2748-2752. doi:10.1182/blood.v95.9.2748.009k31a_2748_2752

Zuber M, Heyden TS, Lajous-Petter AM (1995) A human autoantibody recognizing nuclear matrix-associated nuclear protein localized in dot structures. Biol Cell 85: 77-86. doi:10.1111/j.1768322x.1995.tb00944.x

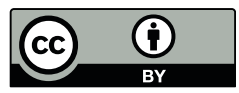

License: This article is available under a Creative Commons License (Attribution 4.0 International, as described at https://creativecommons.org/ licenses/by/4.0/). 